\title{
Review Article \\ WRKY Proteins: Signaling and Regulation of Expression during Abiotic Stress Responses
}

\author{
Aditya Banerjee and Aryadeep Roychoudhury \\ Postgraduate Department of Biotechnology, St. Xavier's College (Autonomous), 30 Mother Teresa Sarani, \\ Kolkata, West Bengal 700016, India
}

Correspondence should be addressed to Aryadeep Roychoudhury; aryadeep.rc@gmail.com

Received 28 December 2014; Revised 3 March 2015; Accepted 7 March 2015

Academic Editor: Shyi Dong Yeh

Copyright ( 2015 A. Banerjee and A. Roychoudhury. This is an open access article distributed under the Creative Commons Attribution License, which permits unrestricted use, distribution, and reproduction in any medium, provided the original work is properly cited.

\begin{abstract}
WRKY proteins are emerging players in plant signaling and have been thoroughly reported to play important roles in plants under biotic stress like pathogen attack. However, recent advances in this field do reveal the enormous significance of these proteins in eliciting responses induced by abiotic stresses. WRKY proteins act as major transcription factors, either as positive or negative regulators. Specific WRKY factors which help in the expression of a cluster of stress-responsive genes are being targeted and genetically modified to induce improved abiotic stress tolerance in plants. The knowledge regarding the signaling cascade leading to the activation of the WRKY proteins, their interaction with other proteins of the signaling pathway, and the downstream genes activated by them are altogether vital for justified targeting of the WRKY genes. WRKY proteins have also been considered to generate tolerance against multiple abiotic stresses with possible roles in mediating a cross talk between abiotic and biotic stress responses. In this review, we have reckoned the diverse signaling pattern and biological functions of WRKY proteins throughout the plant kingdom along with the growing prospects in this field of research.
\end{abstract}

\section{Introduction}

All sustaining living organisms especially the sessile ones like plants are always exposed to a variety of conditions which may cause deleterious impacts on all phenological stages of development. Such adverse conditions are called stress for that particular organism. Harsh environmental conditions which hinder the proper physiological growth of the plant system are called abiotic stresses which include drought, soil salinity, heavy metal, low temperature, radiation, and other forms of oxidative stresses. Adaptation to such environmental stress is essential not only for development of the individual plant, but also for the stability of its successive generations. Genetic manipulation of crop plants has been undertaken to develop stress-resistant crop varieties. The notion of bringing up such genetically modified (GM) crops has become stronger after it was estimated that the maximum worldwide crop yield loss $(70 \%)$ can be attributed to abiotic stress sensitivity of the crops.
Natural evolution in plants has also enhanced multiple level molecular mechanisms to tackle abiotic stress by the induction of stress-responsive and stress-tolerance genes [1]. Such induction is highly dependent on proper perception and transduction of the environmental cues via a signaling cascade [2]. Transcriptional regulation of the stress-induced genes plays a pivotal role in developing stress tolerance in plants. Such regulation is mainly dependent on the temporal and spatial functioning of the transcription factors (TFs) [3]. Presence of 2100 and 2300 TFs has been reported in Arabidopsis thaliana and Oryza sativa, respectively. This shows the enormous importance of TFs in regulating gene expression; otherwise, such a large portion of the genome would not have been devoted for coding the TFs alone [4]. Upregulation of specific TFs corresponding to that of some stress-induced genes has unleashed a complex network of interconnected cross talks. Researchers are trying to find whether specific stress-responsive TFs, apart from upregulating their target genes, also essentially regulate a complete 
package of stress-induced responses like posttranslational and epigenetic modifications, namely, variable nucleosome distribution, histone modification, DNA methylation, and synthesis of nonprotein-coding RNAs (npcRNAs) [5].

\section{TFs in Abiotic Stress}

TFs have been a major target in improving stress tolerance in plants due to their ability to control critical downstream responses by regulating target gene transcription. Entire cascades of signal transduction get activated when TFs interact with specific cis-acting response elements in the promoters of stress-inducible genes. This enhances combinatorial tolerance against multiple stresses [6]. Thus, these TFs themselves can be genetically upregulated to increase the stressinduced gene responsiveness and hence the overall stress tolerance. The principal TFs involved in stress management lie in the AP2/ERF (Apetala2/ethylene response factor), bZIP (basic leucine zipper), NAC (No Apical Meristem, ATAF1/2, Cup-Shaped Cotyledon 2), MYB, C2H2 Zn finger, SBP (Squamosa-Promoter Binding Protein), and WRKY (TFs containing highly conserved WRKY domain) superfamilies [7]. The bZIP TF family consists of a large number of TFs, with diverse roles and ability to bind to abscisic acid- (ABA-) responsive elements (ABREs) [8]. The MYB family of TFs are regulators of several responses pertaining to secondary metabolism, cell cycle, biotic defence, and abiotic stress [9]. The NAC TFs like stress responsive NAC (SNAC) genes when overexpressed improve the drought tolerance in rice [10]. In this review, we will focus on the intricate relation of one of the largest families, that is, the WRKY superfamily of TFs, in enhancing stress tolerance in various plant species.

\section{WRKY Proteins: Structural Characterization and Classification}

The WRKY TFs were first identified in sweet potato (SPF1) as DNA-binding proteins [11]. Evolution of Mutator or Mutatorlike transposable elements (MULEs) gave rise to the WRKYGCM1 superfamily of $\mathrm{Zn}$ finger TFs [12]. The TFs containing the DNA binding domain, GCM, have been clumped together with the WRKY TFs to be classified as the WRKY-GCM1 superfamily [13]. WRKY genes are quite common in plants, though some nonplant species have also been identified which carry such genes in their genomes. 74 WRKY genes in Arabidopsis, more than 100 genes in rice, 197 genes in soybean, 66 genes in papaya, 104 genes in poplar, 68 genes in sorghum, 38 genes in Physcomitrella patens, 35 genes in Selaginella moellendorffi, 80 genes in Pinus, more than 45 genes in barley, 56 genes in Ricinus communis, 119 genes in the B73 inbred line of maize, 55 genes in Cucumis sativus, 120 genes in Gossypium raimondii, and 59 candidate genes in Vitis vinifera have been identified [14-19]. The large number of genes present in the genomes of several plant species does hint about a pivotal role played by these TFs in downstream gene activation. The WRKY domain has a conserved Nterminal sequence of WRKYGQK along with a $\mathrm{Zn}$ finger-like motif, which can be either $\mathrm{Cx}_{4-5} \mathrm{Cx}_{22-23} \mathrm{HxH}$ (C2H2 type) or
$\mathrm{Cx}_{7} \mathrm{Cx}_{23} \mathrm{HxC}$ (C2HC type). The WRKY domain can be 60 amino acids long and binds DNA [20]. Slight variations of WRKYGQK can be found in some WRKY TFs. The WRKY DNA-binding domain generally binds to the $\mathrm{W}$-box elements containing the TTGAC $(\mathrm{C} / \mathrm{T})$ motif, though the flanking sequence adjoining the $\mathrm{W}$-box dictates the binding selectivity of the TF [20]. For example, in contrast to other WRKY TFs, WRKY6 and WRKY11 of Arabidopsis have high affinity towards a $\mathrm{G}$ base upstream of the core motif of the W-box [21].

Arabidopsis being the model plant has the most well classified list of WRKY proteins (Table 1). These proteins have been divided into three distinct groups depending on the numbers of WRKY domains present and the diversity in the $\mathrm{Zn}$ finger motifs found in them [22]. The bifurcation in the groups occurred mainly due to the number of WRKY domains. The proteins belonging to Group I have two distinct WRKY domains, while the proteins belonging to both Groups II and III have single domains. The difference between the proteins of Groups II and III lies in the fact that the former group consists of proteins with the same Cys2His2 $\mathrm{Zn}$ finger motif, while those belonging to the latter group have different Cys2-His/Cys Cys2-His2 Zn finger motif [14]. On the basis of the presence of additional conserved structural motifs, Groups II and III have been further divided into subgroups. Group IV WRKY has been characterized by the loss of the $\mathrm{Zn}$ finger motif. Though these proteins were thought to be nonfunctional, they were found in higher algae (Bathycoccus prasinos) and some plants like rice and Vitis vinifera. Recently, the VvWRKYs from $V$. vinifera have been reported to play crucial roles in generating cold stress tolerance in grapevines (Table 1). Structures like nuclear localization signal (NLS), leucine zippers, Ser/Thr rich stretches, Gln and Pro rich stretches, kinase domains, and pathogenesisrelated TIR-NBS-LRR domains have also been identified in the WRKY proteins which infers the diverse roles played by these proteins in multifarious signaling cascades [14].

\section{WRKY Proteins in Stress}

Under normal cellular conditions, the WRKY genes regulate important functions related to the developmental processes in plants. The expression of the Dactylis glomerata WRKY gene, DGE1, is essential for proper somatic embryogenesis [23]. Strong expression of ScWRKY1 is induced in the fertilized ovules in potato at the late torpedo stage of embryogenesis [24]. Transparent Testa Glabra 2 (TTG2) is also named as WRKY44 and is involved in the regulation of development of trichomes and root hairs [25]. Starch production in endosperm is governed by a WRKY protein, SUSIBA2. High expression of Miniseed 3 gene encoding AtWRKY10 has been reported in pollens, globular embryos, and developing endosperms [25].

The WRKY proteins play prominent roles in the regulation of transcriptional reprogramming associated with plant stress responses (Table 2). Such WRKY-mediated transcriptional response can be against both biotic and abiotic factors [14]. The WRKY proteins function via protein-protein interactions and even cross regulation and autoregulation. 


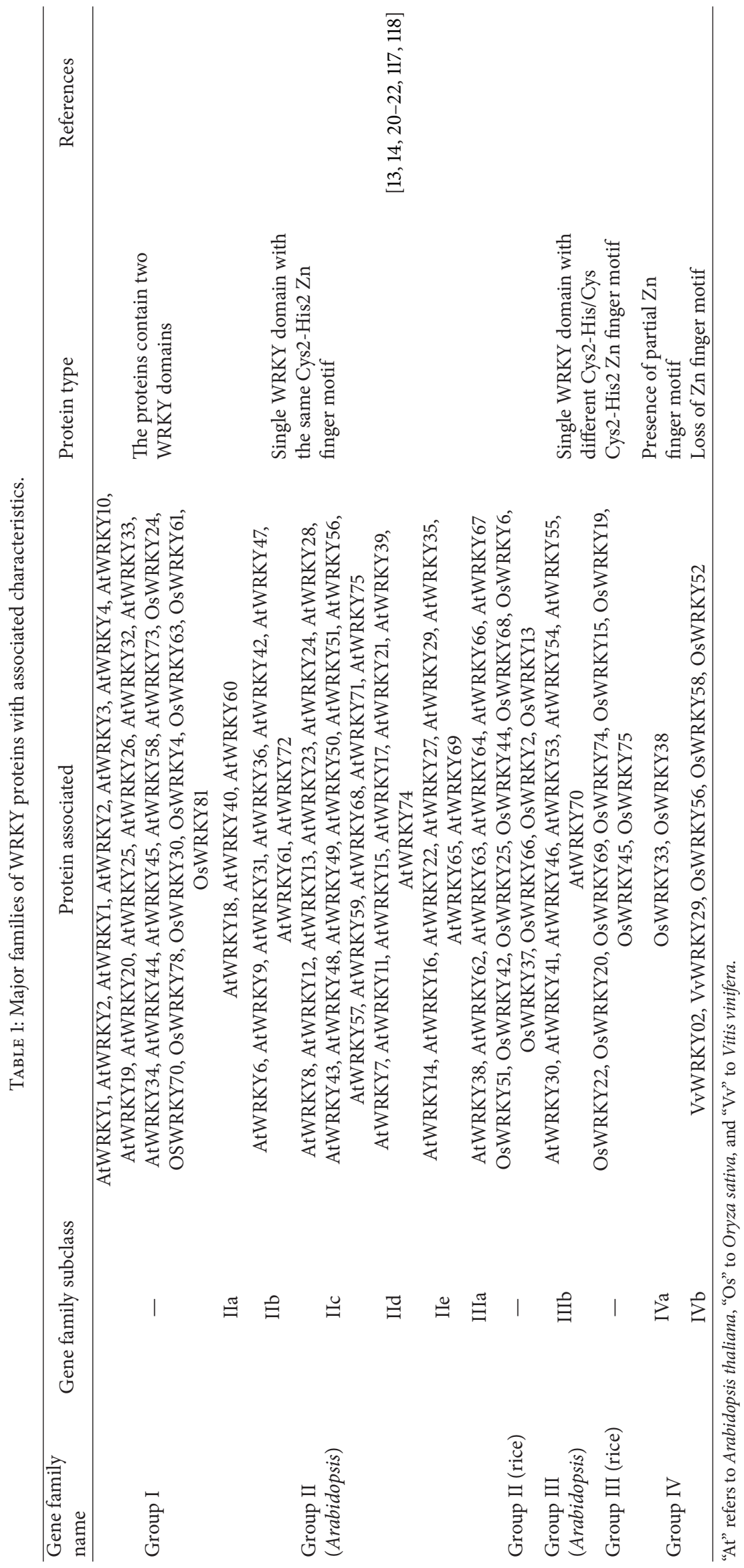




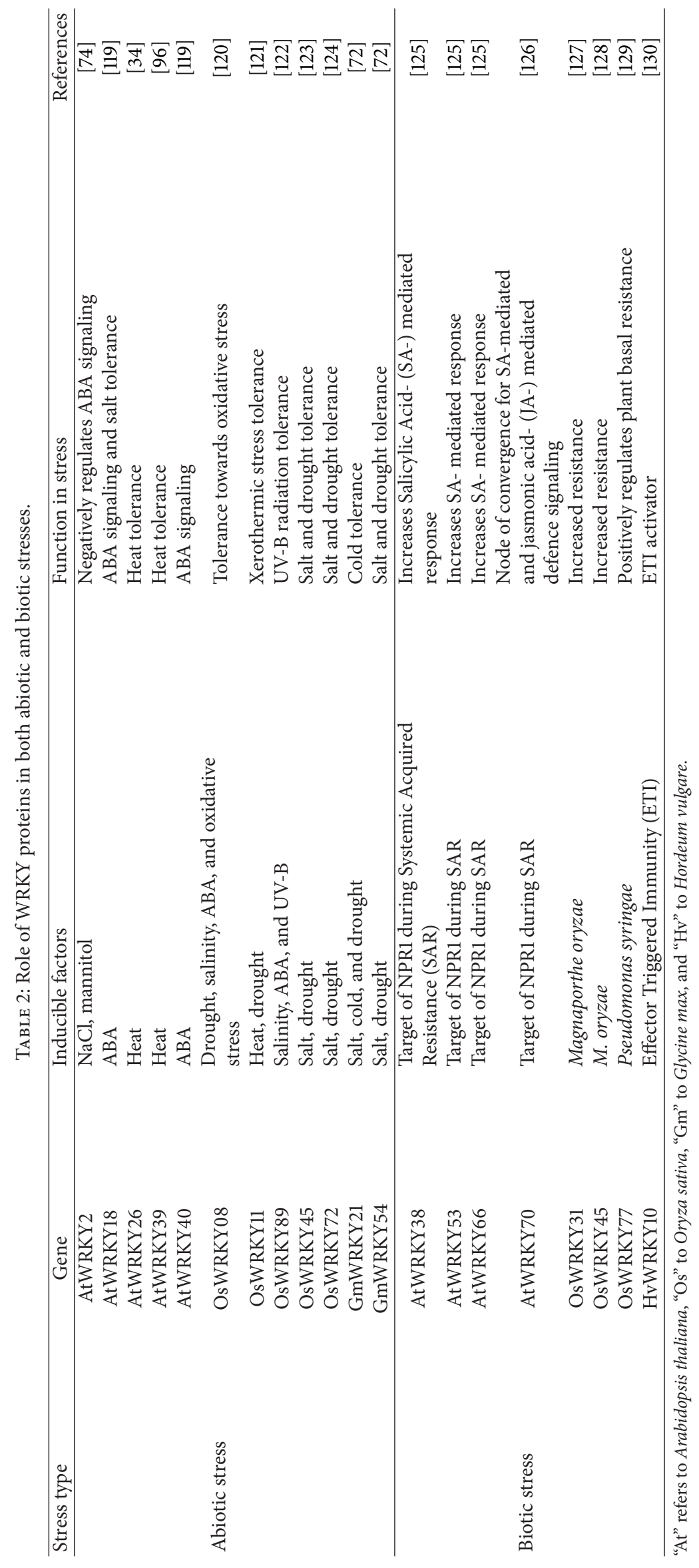


Detailed study on the mechanisms of signaling and transcriptional regulations has unveiled the association of the WRKY proteins with mitogen-activated protein kinases (MAPKs), MAPKKs, 14-3-3 proteins, calmodulin, histone deacetylases, disease-resistance proteins, and other WRKY TFs [26].

Though we will focus on the roles of WRKY in abiotic stress, these proteins have diverse roles in biotic stress as well. Disease resistance and crop yield in the important tropic crop Theobroma cacao have been developed by identifying specific WRKY loci as the genetic markers [27]. In this review, efforts have been made to improve WRKY loci as genetic markers against both abiotic and biotic stresses. Upon designing the complementary PCR primers against the WRKY domains of Group I and Group II (a-c) proteins, 16 WRKY fragments were isolated from a mixture of T. cacao DNA using one pair of primers [27]. Four among these 16 fragments contained single nucleotide polymorphisms within the intron and could be considered as molecular markers after further experimentations [27].

Abiotic stresses like drought, salinity, radiation, and cold induce the activity of several WRKY proteins which function in synchronization to confer resistance against the particular stress or provide a combinatorial effect on multiple stress resistance. Out of the 13 OsWRKY genes, 11 show variable responses towards salt stress, polyethylene glycol (PEG), cold or heat stresses [28]. In wheat, majority (8 out of 15) of the $W R K Y$ genes were transcribed in response to cold, heat, $\mathrm{NaCl}$, and PEG treatment [29]. Induction of 18 AtWRKY genes in the roots of Arabidopsis plants treated with $150 \mathrm{mM}$ $\mathrm{NaCl}$ was confirmed via microarray profiling [30]. The rapid expression of WRKY genes following stress has led to the argument whether such transient increase in WRKY proteins is independent of the de novo synthesis of the regulatory factors [22]. Both activation of adaptive responses and transcriptional regulation of stress-induced genes are actually possible due to the immediate early expression of WRKY genes. Thus, the WRKY protein level in the cell increases sharply and this rise in protein accumulation aids them to regulate target gene transcription by associating with the cis-acting response elements [14]. Table 2 is a concise representation of the role of WRKY genes in stress. However, the table does emphasise on the fact that WRKY TFs mediate tolerance to several abiotic stresses, via transcriptional reprogramming and control of signaling cascades. Expression patterns of WRKY TFs thus have been intricately studied in order to find a proper basis and clue towards overexpressing particular WRKY proteins of choice through transgenic approach.

\section{WRKY-Dependent Signaling Pathways in Abiotic Stress}

5.1. Autoregulation and Cross Regulation. The notion of WRKY proteins involved in critical stress responses obviously makes extensive regulation of the signaling pathway mandatory. In response to both external and internal stimuli, WRKY proteins bind to $\mathrm{W}$-box-containing promoters and trigger the expression of target stress-responsive genes. This triggering is often autoregulated by the WRKY protein itself or by separate WRKY TFs (cross regulation) [26]. Three Group IIa WRKY proteins in Arabidopsis, AtWRKY18, AtWRKY40, and AtWRKY60, have leucine zipper motifs at their Ntermini via which they interact among themselves [31]. The PcWRKY1 of parsley (Petroselinum crispum), apart from binding to its target W-box, also has affinity towards binding the promoters of $P c W R K Y 3$ and even that of the marker gene PcPR1 [32]. The MAPK3/6 activates WRKY33 expression. The WRKY33 proteins in vivo autoregulate their expressions, via a positive feedback loop by binding to their own promoter [33]. Cross regulation among WRKY25, WRKY26, and WRKY33 is essential in promoting tolerance against high temperature stress [34]. The AtWRKY18, AtWRKY40, and AtWRKY60 are directly bound to their respective promoters in order to negatively regulate their expression patterns [13]. The above instances obviously prove the importance of autoregulation and cross regulation in maintaining the homeostasis of WRKY protein expression in the cell.

5.2. Regulation of WRKY Expression by MAPKs. The MAPKs play important roles in transduction of downstream signals in ABA-dependent stress response. Wound-induced protein kinase (WIPK) and salicylic acid- (SA-) induced protein kinase (SIPK) play important roles in biotic stresses like pathogen invasions [35]. The AtMPK3, AtMPK6, and AtMPK4 are activated during both abiotic and biotic stresses [36]. The MAPK cascades phosphorylated OsWRKY30 which enhanced the drought tolerance in rice. Point mutation of Ser in the Ser-Pro (SP) site resulted in a drought-sensitive crop [37]. This illustrates the crucial role played by MAPK phosphorylation in proper OsWRKY30 activity (Figure 1). Recent reports have characterized the presence of two pollenspecific WRKY TFs (WRKY34 and WRKY2) during male gametogenesis in Arabidopsis thaliana. Overexpression of WRKY34 using a strong pollen-specific promoter led to the phosphorylation of the WRKY34 protein by MPK6 and MPK3 [38]. Mutations in the phosphorylation sites in WRKY34 compromised its functions in vivo [38]. In vivo phosphorylations of WRKY TFs by MAPKs have also been recently reported [39]. The MPK3/MPK6 cascade along with the downstream WRKY TF has been depicted to induce ethylene production through regulation of ACC synthase activity [40]. The MAPK cascades involved in phosphorylating WRKYs involved in abiotic stress are less studied in comparison to the biotic counterparts. However, the knowledge of these signaling cues can impose further improvements in designing stress-tolerant transgenic crops.

\section{Interaction between WRKY TFs and Associated Factors in Abiotic Stress}

6.1. VQ Proteins. VQ proteins are a group of cofactors containing a short VQ-related motif (FxxxVQxLTG). Out of the $34 V Q$ genes reported in Arabidopsis, the majority do not contain any intron and encode small proteins of 100200 amino acid residues having the consensus VQ motif. It has been reported that these $34 \mathrm{VQ}$ proteins can interact with WRKY TFs in yeast [41]. The VQ proteins are often 


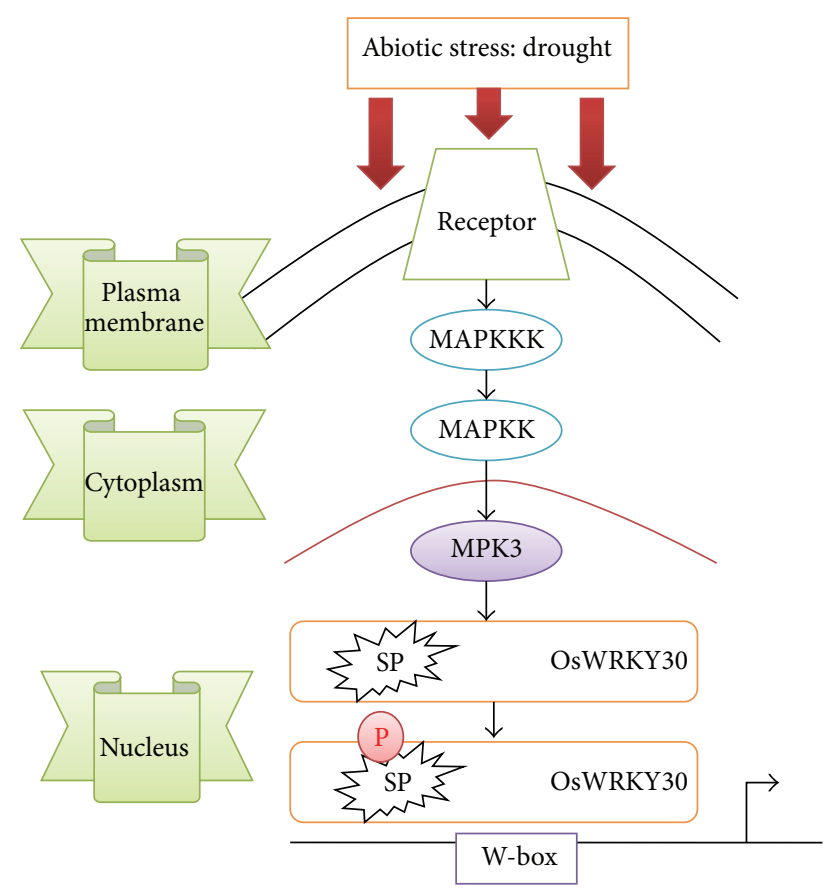

FIGURE 1: The mitogen-activated protein kinase (MAPK) pathway induces the activity of OsWRKY30 during drought stress. Stress signals are sensed via a transmembrane receptor, which with the help of some unknown molecules and adaptor proteins activates the MPK/MAPK pathway. This leads to the phosphorylation and activation of the MPK3. MPK3 phosphorylates the target Ser residue in the SP motif of OsWRKY30 and activates the same. The activated WRKY protein then undergoes a conformational change which favourably allows it to bind to the $\mathrm{W}$-box of its target gene to induce transcription. The protein product encoded by the target gene probably helps the plant system in combating the drought stress.

activated by MAPK cascades in response to stress signal cues. During post-association with MAPK substratel (MKS1), VQ protein interacts with AtWRKY33 and AtWRKY25 to act as a substrate of MAPK4 [42]. Interaction of VQ proteins with WRKY TFs results in stimulation or inhibition of the latter to bind to its specific DNA. This mainly occurs because VQ protein binding manipulates the WRKY TF to change its preference for the nucleotides flanking the conserved W-box [43]. The resulting alteration in target gene specificity of the WRKY TF gives rise to a changed biological downstream response. Diversification of WRKY TF-induced responses may also result from interactions with multiple VQ proteins [43]. Tolerance to multiple abiotic stresses occurred in Arabidopsis when the WRKY33 interacted with multiple VQ proteins including Sigma Factor-Interacting Protein1 (SIB1) and SIB2, via the C-terminal of the WRKY domain. This interaction induced the DNA binding activity of AtWRKY33 [44]. SIB1 and SIB2 have been assumed to play roles in regulation of transcription and retrograde signaling from chloroplast and mitochondria to the nucleus. VQ proteins have also been hypothesised to induce chromatin remodelling as an abiotic stress response [44]. MVQ1 is a VQ-motif-containing protein which was recently depicted to control WRKY-regulated defense gene expression [45].

6.2. Histone Modifying Chromatin Remodelling Complex. A condensed chromatin structure wrapped within the nucleosomal complex in association with histone proteins and other packaging factors does not easily interact with the transcriptional machinery. So, when the plant is not under stress, the gene remains transcriptionally silent. The sensing of environmental cues signals the WRKY TFs to induce the transcription of target stress-inducible genes. The packed chromatin structure has to be loosened to facilitate proper association of the transcription complex at the promoter site. Thus, it is often considered that such chromatin remodelling complex, along with autoregulation and cross regulation, plays a crucial role in WRKY TF-induced responses. AtWRKY70 was reported to be stimulated by Arabidopsis homolog of trithorax (ATX1) and, as a result, the nucleosomal histone H3K4 trimethylation occurred [46]. Epigenetic regulation by histone methyltransferase is endowed upon AtWRKY53, a senescence regulator. Activation of AtWRKY53 in response to senescence triggered the rise in $\mathrm{H} 3 \mathrm{~K} 4$ dimethylation and $\mathrm{H} 3 \mathrm{~K} 4$ trimethylation at the $5^{\prime}$ end and coding regions of WRKY53 [47]. In Musa acuminata, the protein encoded by the linker histone H1 gene (H1S1) interacted with MaWRKY1 in response to the stress caused by the postharvest ripening of fruits induced by ethylene. The induction of MaH1S1 is also accelerated in presence of jasmonic acid (JA), ABA, and hydrogen peroxide and under chilling stress [48]. Overexpression of AtWRKY38 and AtWRKY62 enhanced the resistance of the plants to pathogenic attack by Pseudomonas syringae. It has been reported that the proteins encoded by these genes interact with Histone Deacetylase 19 (HD19). Overexpression of HD19 retarded the activities of AtWRKY38 and AtWRKY62 as TFs [49].

6.3. Calmodulin and 14-3-3 Proteins. The WRKY proteins have a calmodulin (CaM) binding domain (CaBD). Site directed mutagenesis studies have confirmed the importance of this domain in WRKY TFs to bind CaM [43]. The AtWRKY7 associated with CaM through its own CaBD. The AtWRKY7 CaBD consists of VAVNSFKKVISLLGRSR. Ten other Arabidopsis Group IId WRKY proteins have been found to possess such related CaBDs (DxxVxKFKxVISLLxxxR). Thus, these proteins also have a tendency to interact with CaMs [50]. In case of overlapping WRKY-WRKY interactions, the steric hindrance prefers WRKY-CaM interaction, provided that the calcium concentration in the cell is high [43].

Seven WRKY proteins in Arabidopsis have been identified via proteomic profiling of tandem affinity-purified 14-3-3 complexes to interact with $14-3-3$ proteins [51]. Out of these, AtWRKY6 is induced under phosphate starvations. AtWRKY18 and AtWRKY40, complexed with 14-3-3 proteins, participate in ABA signaling [52]. WRKY proteins interacting with 14-3-3 proteins are subjected to phosphorylation by the latter in response to stress-activated signaling cascades [53]. The 14-3-3 proteins are also capable of dimerization and each dimer binds two substrates. Thus, 
WRKY proteins with phosphorylated binding sites have the tendency to associate with other factors and proteins by mutual interactions with true 14-3-3 dimers. This is the case of indirect association of WRKY with other proteins in the complex $[43,54]$. Further studies are required which will aid in developing the database of dynamic interactions of 14-33 proteins with WRKY TFs in the spatiotemporal context of abiotic stress signaling cascades.

\section{Cross Talk between WRKY TF and ABA-Mediated Signaling}

WRKY superfamily of TFs is the major regulator in plant defence and SA-mediated signaling. However, significant instances are there which show that these TFs also participate in $\mathrm{ABA}$-mediated signaling [55]. ABA is the universal stress hormone and the WRKY proteins associated with ABA signaling can definitely influence stress-induced responses. AtWRKY40 has been characterised as a negative regulator of ABA signaling during seed germination. AtWRKY40 also interacts with AtWRKY18 and AtWRKY60 to inhibit the expression of crucial stress-responsive genes [52]. WRKY18 and WRKY60 interact with the W-box of the downstream $A B A$ Insensitive genes like $A B I 4$ and $A B I 5$ in order to repress their expression [56]. Utilising a stable transgenic reporter or effector system, it was observed that WRKY18 and WRKY60 act as weak transcriptional activators, while WRKY40 is a transcriptional repressor in plant cells [57]. WRKY63 in Arabidopsis is responsible for enhanced drought tolerance. Increased $A B A$ sensitivity and reduced drought tolerance were observed when the WRKY63/ABA Overly Sensitive3 (ABO3) was disrupted. The drought tolerance reduced especially due to unresponsive ABA-induced stomatal closure. AtWRKY63 binds to the promoters of ABA Responsive Element Binding Proteins/Factors (AREB1/ABF2) [13]. Reports on the relation between $A B A$ and abiotic stress-mediated WRKY genes have identified 16 ABA-related WRKY genes in rice. 12 of them were seen to have higher expression in response to cold, drought, and salinity [58]. A study showed that the cross talk between Gibberellic Acid (GA) and ABA is mediated by OsWRKY51 and OsWRKY71 in rice. The expression of the genes encoding these two WRKY proteins is induced by ABA, and this results in a high ratio of OsWRKY51/OsWRKY71 repressors to GAMYB activator [59]. GA induction of $\alpha$ amylase promoter $(A m y 32 b)$ is suppressed due to the binding of OsWRKY51/OsWRKY71 repressors with the respective W-boxes. In such a situation, GA induces the production of GAMYB and inhibits OsWRKY51 and OsWRKY71. Due to higher levels of GAMYB, the $\alpha$-amylase gene expression increases [59]. Other activators of $\alpha$-amylase gene expression via GA are protein factors like OsDOF3, RAMY, and OsMYBs1/OsMYBS2. The repressors other than those mentioned above are KGM and HRT [59, 60].

Larrea tridentata (creosote bush) due to its xerophytic evergreen nature has very high tolerance towards drought. WRKY21 of 314 amino acid residues with localization in the nucleus has been isolated from the creosote bush. LtWRKY21 binds to the promoter of ABA-responsive gene HVA22 and promotes transcription of the same. This activation of transcription is dependent on the levels of LtWRKY21 [61]. The HVA22 protein has important roles in combating multiple abiotic stresses. High gene expression occurs due to coexpression of activators like $V P 1$ and $A B A$ Insensitive 5 (ABI5), along with $L t W R K Y 21$. The dominant negative mutant protein phosphatases like abil-1 are not inhibitors of LtWRKY21, $V P 1$, and ABI5 coexpression, though these mutant phosphatases are negative regulators of $A B A$ signaling. This proves the fact that the complex of LtWRKY21, VP1, and ABI5 obviously regulates downstream of ABI1 in ABA-mediated response cascades during abiotic stress [61]. AtWRKY57 has shown enhanced expression in response to higher ABA levels conferring higher drought tolerance to the plant [62]. Chromatin immunoprecipitation (ChIP) assays confirmed the binding of WRKY57 to the W-box of Responsive to Desiccation 29A (RD29A) and 9-cis-epoxycarotenoid dioxygenase 3 (NCED3) promoters. Thus, WRKY57 functions by directly inducing stress-responsive genes [62]. In rice, OsWRKY451 and OsWRKY45-2 participate in ABA-mediated responses during abiotic stress. OsWRKY45-2 had a negative effect on ABA-induced downstream responses to salt stress. These two WRKY proteins have also been proved to regulate ABAdependent signaling during drought and low temperature stresses [63]. WRKY proteins can act both as activators and repressors to ABA-inducible promoters. OsWRKY24 and OsWRKY45 act as the repressors, while OsWRKY72 and OsWRKY77 act as the activators [64]. WRKY TFs have also been reported to upregulate ABA-responsive genes like ABF4, ABI4, MYB2, Dehydration Response Element Binding Protein 1a (DREBP1a), DREBP2a, and Response to ABA 18 (RAB18). Several WRKY TFs have been reported to be positive regulators of ABA-mediated stomatal closure, while some are negative regulators of seed germination and also indirectly control flowering [65]. In ABA-treated, salttolerant rice variety Pokkali, the expression of WRKY71 increased, while feeble induction was reported for WRKY24 [66]. Recent findings showed that the expression of WRKY8 was downregulated by the crucifer-infecting tobacco mosaic virus (TMV-cg). In the systemically infected leaves of the wrky 8 mutants, the expression of ABI4 was reduced, while that of 1-aminocyclopropane-1-carboxylic acid synthase 6 (ACS6) and the ethylene response factor 104 (ERF104) was enhanced. The accumulation of TMV-cg was reduced on exogenous application of ABA [67]. WRKY20 isolated from Glycine soja was characterized to regulate ABA signaling and enhance drought tolerance. The GsWRKY20 has also been reported to be associated with flowering with high expression in the shoot tips and the inflorescence meristems of wild soybean [68].

\section{Transgenic Approaches for Overexpression of WRKY Proteins during Abiotic Stress}

8.1. Drought and Salinity Stresses. Prolonged drought in a particular area results in a physically dry soil which is unsuitable for crop productivity. On the other hand, high salt concentration gives rise to a physiologically dry soil 


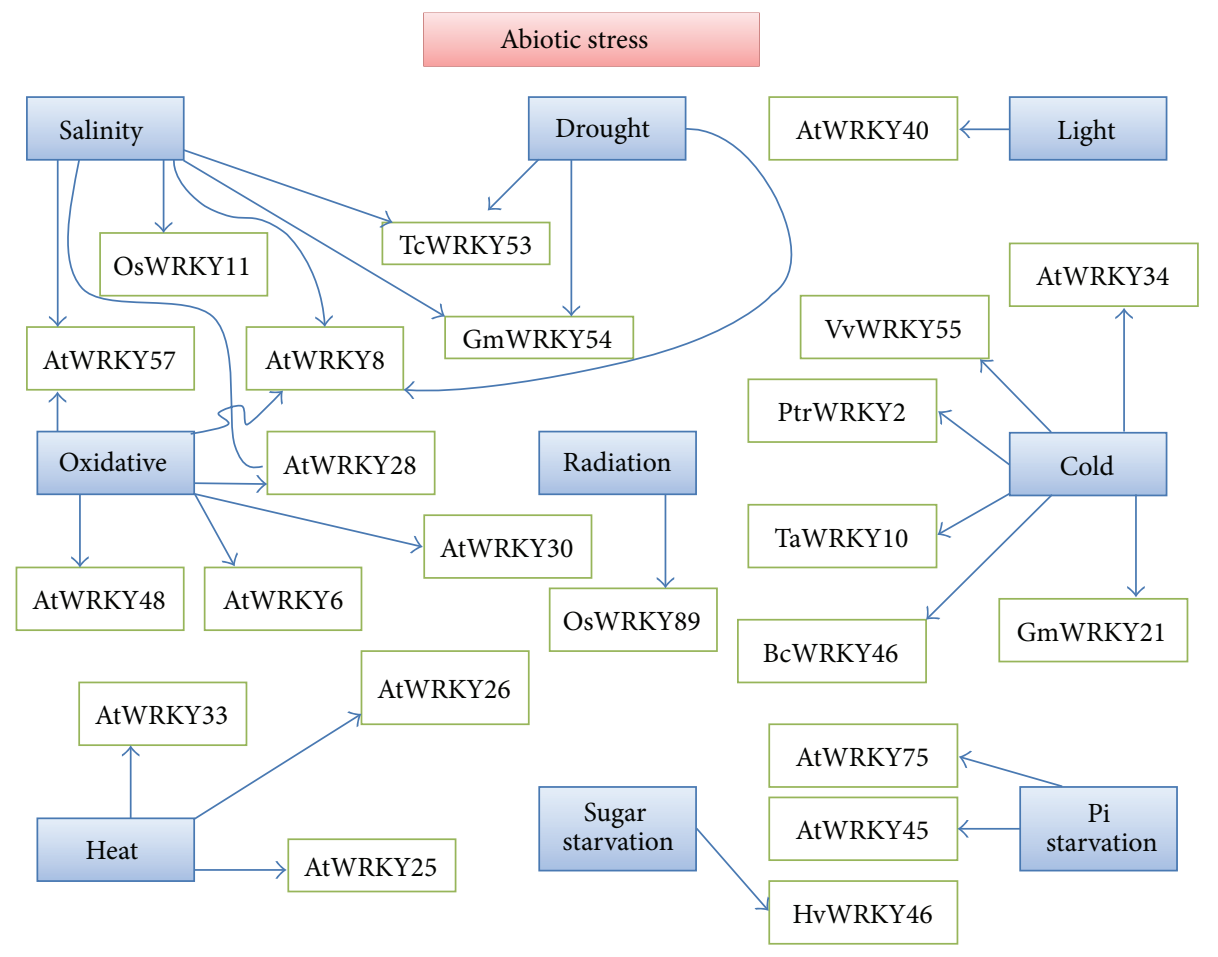

FIGURE 2: WRKY proteins regulating plant responses against multiple abiotic stresses like salinity, drought, heat, cold, nutrient starvation, light, radiation, and oxidative stresses. "At" refers to Arabidopsis thaliana, "Os" refers to Oryza sativa, "Gm" refers to Glycine max, "Vv" refers to Vitis vinifera, "Hv" refers to Hordeum vulgare, "Ta" refers to Triticum aestivum, "Bc" refers to Brassica campestris, and "Ptr" refers to Poncirus trifoliata.

which is also antagonistic for sustainability of the crops. Thus, drought and salinity stresses merge at a common point of water shortage, thus inhibiting crop productivity. This is the reason for which most of the WRKY proteinmediated responses for both stresses are very common in nature. Since the response is overlapping, it becomes difficult to divide the WRKY proteins into a particular group responsible for drought tolerance and another responsible for salt tolerance [69]. Under the control of HSP101 promoter, the overexpression of OsWRKY11 resulted in lower rates of leaf-wilting and enhanced chlorophyll stability, along with sustenance of the green parts. These factors help in generating higher drought tolerance in the crop [70] (Figure 2). The 35S: OsWRKY45 and 35S:OsWRKY72 Arabidopsis plants had higher expression of the ABA-inducible genes which aided in developing higher salt and drought tolerance in the plant [71]. Overexpression of GmWRKY54 from Glycine max in transgenic lines enhanced the salt and drought tolerance (Figure 2). High levels of GmWRKY54 are thought to manipulate the expression of the TF gene, salt tolerance Zn finger (STZ/Zat10), and DREB2A. The transgenic plants overexpressing the GmWRKY13 had decreased sensitivity to ABA, while these plants had less tolerance towards high salt and mannitol in comparison to the wild types. Thus, it can be inferred that GmWRKY13 is a negative regulator of abiotic stress response when overexpressed alone [72]. Exposure to salt and drought stress induced the expression of TcWRKY53 in Thlaspi caerulescens. Two ethylene response factor (ERF) family genes in tobacco, NtERF5 and NtEREBP-1, had low transcription levels in transgenic tobacco plants overexpressing TcWRKY53 [73]. The expression of the Late Embryogenesis Abundant (LEA) family gene NtLEA5 remained unaffected, indicating the fact that TcWRKY53 increases osmotic stress tolerance through interaction with an ERFtype TF. It is probable that TcWRKY53 does not directly manipulate the stress-responsive genes [73]. Overexpression of either AtWRKY25 or AtWRKY33 conferred salt tolerance in Arabidopsis. These two WRKY proteins are closely related and their transcript levels subsequently increased when the plants were treated with high concentrations of salt. The importance of these proteins was proved from a mutation experiment considering Atwrky33 null mutants and double mutants of Atwrky25 and Atwrky33. Both types of mutants exhibited increased sensitivity to saline stress [34]. Arrest of seed germination under the influence of ABA is levied by AtWRKY2, which has been proposed to act as a negative feedback regulator of ABA-induced seed dormancy [74]. AtWRKY57, AtWRKY8, and AtWRKY28 regulate signaling cascades in salinity, drought, osmotic, and oxidative stresses [75] (Figure 2).

Results furnished by suitable experiments showed that eight out of 15 WRKY genes in wheat are induced by high salt concentrations, PEG, cold, or heat [29]. Overexpression of OsWRKY30 activated by a MAPK cascade enhanced the tolerance of transgenic rice to drought [40]. The salt and drought tolerance dramatically increased when TaWRKY10 
from wheat was introduced and overexpressed in tobacco. TaWRKY10 has been depicted as a major TF, activating multiple stress-related genes, and also has a role in maintaining the osmotic balance in the cell. The transgenic tobacco lines exhibited remarkably high levels of proline and soluble sugar but low levels of malondialdehyde (MDA) when exposed to drought or salt stress [76]. TaWRKY2 and TaWRKY19 overexpression in Arabidopsis led to more efficient salt, drought, and low temperature tolerance. The overexpression of BcWRKY46 and HvWRKY38 in Arabidopsis resulted in enhanced tolerance towards drought and salt stresses [76]. The protein products of these genes are nuclear proteins and act as TFs, targeting many downstream genes which need to be activated to combat abiotic stress [77]. Exposure of Arabidopsis to salinity stress resulted in twofold increase in the levels of 18 WRKY transcripts and repression of eight WRKY genes [58]. We have already discussed the induction of ABA-responsive genes due to the AtWRKY40ABAR (ABA-binding protein) interaction. AtWRKY18 and AtWRKY60 act in synchronization to increase the sensitivity of the plant towards salt and osmotic stresses [14]. The expression of PtrWRKY2 gene in Poncirus trifoliata was suppressed by $27-50 \%$ upon exposure to prolonged drought stress. However, in drought-stressed Citrus maxima (pummelo) plants, the expression pattern of PtrWRKY2 remained unaltered [78]. Salt and ABA treatment increased the transcript levels of OsWRKY08. Osmotic stress tolerance via positive regulation of two ABA-dependent genes like AtCOR47 and AtRD21 was reported in transgenic Arabidopsis overexpressing OsWRKY08 [24]. Recent reports highlighted the upregulation of AtWRKY46 during osmotic stresses like salinity and drought. The roles of WRKY46 in mediating cellular osmoprotection and redox homeostasis under stress have also been depicted via microarray analysis. Regulation of light-dependent starch metabolism is performed by WRKY46 through the control of the QUA-QUINE STARCH (QQS) gene expression [79]. The group II family of WRKY TFs (JcWRKY) found in the biofuel crop Jatropha curcas developed tolerance against ionic, osmotic, and chemical stresses when expressed in E. coli. Transcript analysis showed that transcription of $J c W R K Y$ was increased in response to salinity, dehydration, salicylic acid, methyl jasmonate, and the collar rot fungus Macrophomina [80]. In cotton (Gossypium hirsutum), a Group IId WRKY gene GhWRKY17 was found to be associated with salt and drought stress. Increased drought and salt sensitivity resulted in transgenic tobacco plants (Nicotiana benthamiana) overexpressing GhWRKY17. GhWRKY17 lowered ABA sensitivity leading to low transcription of ABA-inducible genes like $A R E B$, $D R E B, N C E D, E R D$, and LEA [81]. These instances obviously indicate the enormous influence of the WRKY proteins in activating a proper response against salt and dehydration. The plants which appear to be tolerant under these harmful environmental circumstances show persistence of green parts. This has been obviously due to shielding of chlorophyll and other necessary pigments required for photosynthesis. Thus, the WRKY TFs must be playing crucial roles in guarding these pigments against the low water status of the cell and preventing influx of salt beyond the threshold limits.
The content of Reactive Oxygen Species (ROS) in the tissues of the tolerant varieties is also low [82, 83]. 61 WRKY genes were identified in Populus which were induced by both abiotic and biotic treatments like infection with Marssonina brunnea, SA, methyl jasmonate, wounding, cold, and salinity. 46 genes from this cluster were shown to be expressed in roots, stems, and leaves [84]. These results probably hint towards the role of WRKY TFs as activators of genes encoding LEA proteins like dehydrins or even some genes in the biosynthetic pathways of compatible solutes like proline, polyamines, and so forth.

8.2. Oxidative Stress. WRKY has often been depicted as an aggravator of ROS production in cells. The ROS like superoxide, hydroxyl radicals, and hydrogen peroxide have tremendous negative impact on the concerned cell wall leading to lipid peroxidation, cell damage, and oxidative stress. The uptake of oxygen is responsible for such oxidative burst of ROS [82, 85]. Hydrogen peroxide treatment in Arabidopsis triggered higher expression of AtWRKY30, AtWRKY75, AtWRKY48, AtWRKY39, AtWRKY6, AtWRKY53, AtWRKY22, and AtWRKY08 [86] (Figure 2). The WRKY proteins also help in quenching the ROS produced in mitochondria. The changes in environmental conditions and retrograde signaling affect the expression of several nuclear genes encoding mitochondrial proteins. About 72 WRKY proteins in Arabidopsis genome have been proved to regulate production of nuclear transcripts encoding mitochondrial proteins. These WRKY proteins do have WRKY domains complementary to the W-box at the promoters on the nuclear transcripts [87]. WRKY binds to the promoters of marker genes like Alternative oxidasela (AOX1a), NADH dehydrogenase B2, and the AAA ATPase Ubiquinol-cytochrome c reductase synthesisl. The effects of antimycin A-induced mitochondrial retrograde expression and high light-induced stress were reduced by the overexpression of AtWRKY40 [88]. On the contrary, AtWRKY63 acts as an activator in inducing high light stress tolerance. It can be assumed that high lightinduced stress leads to the formation of ROS which can be somehow quenched by downstream responses triggered by AtWRKY63. Coordination in the coding of stress-responsive genes in mitochondria and chloroplast has been studied through the functions of AtWRKY40 and AtWRKY63. These proteins regulate the expression of stress-responsive genes common to both mitochondria and chloroplasts without disturbing the constitutive expression of the house-keeping genes in other organelles [87]. Another instance of WRKY proteins involved in oxidative and light stress is the T-DNA knockout mutant of APX1 gene which led to the induction of AtWRKY6, AtWRKY18, AtWRKY25, AtWRKY33, AtWRKY40, AtWRKY46, AtWRKY54, AtWRKY60, and AtWRKY70 [13]. AtWRKY70 exhibited constitutive expression in the Atapxl mutants [89]. Thus, WRKY proteins indirectly do help in the scavenging of ROS in order to alleviate oxidative stress.

The key proponents of the ROS signaling cascade are ascorbate peroxidases (APX), NADPH oxidases, and Zn finger proteins. Researchers have proposed the relation between Zn finger proteins and WRKY factors in alleviating ROS 
toxicity. AtWRKY25 could not be formed in appropriate amounts in Atzat12 mutant (gene for Zn finger protein) plants exposed to high levels of hydrogen peroxide. Thus, it can be obviously suggested that the expression of AtWRKY25 is dependent on the protein encoded by AtZat12 [90]. The TF TaWRKY10 of wheat when overexpressed in transgenic tobacco decreased the accumulation of MDA and lowered the levels of superoxide radical and hydrogen peroxide formation on exposure to salinity and drought stresses. Low MDA was attributed to low rates of lipid peroxidation. The transgenic seedlings showed increased tolerance towards oxidative stress due to higher accumulation of TaWRKY10 [77]. The transgenic tobacco plants overexpressing GhWRKY17 exhibited higher sensitivity towards oxidative stress. The expression of the genes for ROS-scavenging enzymes like APX, catalase (CAT), and Superoxide Dismutase (SOD) was suppressed in the transgenic lines [81]. The Arabidopsis lines overexpressing the TFs, Helix Loop Helix17 (HLH17) and WRKY28, showed enhanced tolerance towards osmotic stress [75]. WRKY30 was rapidly expressed as a primary response to hydrogen peroxide and methyl viologen (MV). MV acts as a superoxide anion propagator in light [91]. Transgenic Arabidopsis plants overexpressing WRKY15 were more sensitive to both salinity and oxidative stresses with the formation of increased leaf area and accumulation of increased plant biomass. WRKY15 induced leaf area increment through intensified endoreplication and not by extending the cell numbers. WRKY15 expressed under oxidative stress aids in the activation of mitochondrial genes which code for proteins belonging to the family of mitochondrial dysfunction regulon. WRKY15 has also been proposed as a general repressor of genes whose products participate in mitochondrial retrograde signaling [92]. During dark-induced senescence, the leaves of Pelargonium cuttings showed high accumulation of WRKY6. The basis of an increment in ROS level together with higher expression of senescence-associated protease homologs PeSAG12-1 and PeWRKY6-1 could not be explained [93]. OsWRKY42 has been portrayed as a negative regulator in oxidative stress. This is because overexpression of OsWRKY42 in rice resulted in high accumulation of ROS. It was also reported that OsWRKY42 binds to the W-box of the OsMT1d (rice Metallothionein 1d) gene and represses its expression, thereby promoting leaf senescence [94]. TaWRKY10 gene isolated from Triticum aestivum was reported to be a positive mediator of plant tolerance against salinity and drought through the regulation of osmotic balance, ROS scavenging, and transcription of stress-related genes [95].

Osmotic and oxidative stresses are linked at a point as both decrease viability of crops in general. This cross talk in the signaling cascades of the plant in developing tolerance against varied abiotic stresses has obviously opened several new avenues of research. One of them is to genetically modify some particular target components in the plant system in order to design a transgenic crop which can be tolerant to multiple abiotic stresses. Our discussion gives a clear indication of the fact that WRKY proteins are emerging players in generating abiotic stress tolerance in crops. Though this group of TFs are more related to biotic stresses like pathogen attack and plant defence, their immensely growing significance in response to abiotic stress cannot be neglected at all.

8.3. Temperature Stress. Physiological processes in a plant system are best operated at an optimum temperature. Most of these pathways are dependent on enzymes, which have maximum activity at an optimum temperature. Beyond this limit, the activity of the enzymes gradually decreases as the protein structures are affected and ultimately the entire pathway comes to a halt. This is true for temperatures which are either higher or lower than the optimum operating temperature of the system. Several researches have been undertaken to study the growth patterns in crops exposed to extremes of temperature. This has revealed the important roles played by WRKY proteins in response to such stresses.

Tolerance to heat stress has been depicted to be regulated by the Group I WRKY proteins, AtWRKY25, AtWRKY26, and AtWRKY33 (Figure 2). When the Arabidopsis plant is exposed to high temperature stress, the expression of AtWRKY33 is repressed, while that of AtWRKY25 and AtWRKY26 is stimulated. Inhibited seed germination, lower survival, and electrolytic leakage were seen in the heatstressed plants having mutations at the above three loci. On the contrary, increased tolerance towards heat stress was recorded in transgenics overexpressing AtWRKY25, AtWRKY26, and AtWRKY33 [34]. The fact that these genes are activated by a heat-induced ethylene-dependent response has raised the probability of possible convergence and cross talk between the ethylene signaling pathways and the signaling cascades activated in response to heat stress [34]. A possible cross talk between biotic and abiotic stress response components has also been noted in case of AtWRKY39. The Arabidopsis gene AtWRKY39 is induced in response to heat stress and the WRKY protein encoded by this gene positively regulated the interaction between the SA and JA signaling cascades [96]. Heat stress has been found to regulate about nine of the 60 analyzed WRKY genes. Out of these nine genes, AtWRKY7 has been identified as a Heat Shock Factor Ala/1b (HsfAla/1b), triggering the heat stress-induced genes [97]. We have previously discussed that overexpression of OsWRKY11 under the control of HSP101 promoter led to increased drought tolerance. It was reported that these transgenic plants evolved tolerance towards high temperature stress as well [70].

AtWRKY34 expression is stimulated in response to pollen-specific cold stress. AtWRKY34 has been proved to be a crucial locus in cold stress regulation as overexpression of AtWRKY34 makes the pollens sterile even under normal growth conditions [98]. The mutation of this gene enhances the cold stress tolerance in the pollens. Thus, AtWRKY34 negatively regulates the development of cold stress tolerance in pollens. This regulation is achieved by proper manipulation of the transcriptional activators, namely, C-repeat Binding Factors (CBFs) [99]. Increased tolerance to cold stress has also been reported in transgenic Arabidopsis overexpressing GmWRKY21 [65]. Transgenic plants overexpressing TaWRKY10 also showed enhanced cold stress tolerance [77]. The Poncirus trifoliata WRKY gene PtrWRKY2 is an 
important player in cold stress (Figure 2). The expression of this gene increased initially when both cold-tolerant Poncirus and cold-sensitive pummelo were exposed to cold stress. However, the gene expression subsided in both cold-tolerant Poncirus and cold-sensitive pummelo after exposure to 1 hour and 1 day of cold stress, respectively [78]. HvWRKY38 has a role in freezing tolerance as the expression of the corresponding gene was strongly stimulated when the plants were constantly subjected to freezing temperatures [99]. Similarly, PtrWRKY2 may also have some role in developing freezing tolerance in Poncirus or pummelo. In rice, 41 out of 103 WRKY genes exhibited variable expression patterns in response to chilling stress [58]. 25 WRKY genes in Glycine max showed differential transcription patterns in response to cold [72]. In Vitis vinifera (grapevine), majority of the $59 \operatorname{VvWRKY}$ genes were expressed in tissues of young and mature leaves, tendrils, stem apex, roots, and young and ripened fruits [100]. The gene-chip based data was analysed and it was reported that $36 V v W R K Y$ genes had their expressions increased by twofold on exposure to cold. Phylogenetic studies have confirmed the possibility of a cross talk between stress responses to salt, PEG, and colddependent VvWRKYs. The VpWRKY3 is a homologous gene of $V v W R K Y 55$. On exposure to cold, the levels of VpWRKY3 steadily increased in the cell, while VvWRKY55 expression was upregulated under extremely low temperatures [100]. The expression of $V v W R K Y 43$ increased in Solanum dulcamara in the colder months [101]. In the roots of barley exposed to cold, transient increase in the expression of WRKY38 occurred. Cold stressed Pak-choi had their BcWRKY46 genes upregulated. Transgenic tobacco plants which exhibited constitutive expression of BcWRKY46 were more tolerant to cold stress in comparison to the wild type plants [102]. The expression of WRKY71 in banana peaked when the plant was treated under extremely low temperature conditions [103]. Antagonistic effects in relation to abiotic and biotic stresses were seen in rice plants with overexpressed OsWRKY76. In such plants, a specific set of Pathogenesis related (PR) genes and other genes involved in phytoalexin synthesis, after inoculation with blast fungus, were downregulated, while the cold stressassociated genes like peroxidase and lipid metabolism genes were upregulated [104].

8.4. Stress due to Deficiency of Nutrients. Since plants are sessile organisms, they are completely dependent on the nutrients and essential elements present in their rhizosphere. Deficiency in an important element is strongly antagonistic to proper plant development. This also leads to impairment of multiple physiological pathways as they are always linked at some level or through a common intermediate or cofactor. Salinity or drought is actually a condition related with the characteristics of soil components. In salinity, the $\mathrm{NaCl}$ content of the soil surpasses the threshold level to sustain plant growth, while, in drought, the moisture content of the soil is not enough to support germination or development. Nutrient deficiency also falls in the same brackets as salinity and drought. This is because nutrient deficiency is also soilrelated.
WRKY TFs are major regulators in overcoming the adversities related to nutrient deficiency in plants grown on nutrient deficient soil (Figure 2). The first WRKY protein involved in nutrient deficiency was AtWRKY75. The gene encoding AtWRKY75 exhibited stimulated expression under phosphate $(\mathrm{Pi})$ deficient conditions. Mutations resulting in silencing of AtWRKY75 showed higher plant sensitivity towards Pi stress accompanied with reduced absorption of Pi. The AtWRKY75 RNAi plants had reduced expression of Pi starvation-induced genes like phosphatases, Mt4/TPS1 like genes, and transporters with high affinity for transporting $\mathrm{Pi}$ [105]. A negative regulator of Phosphate 1 (PHO1) expression in Arabidopsis is the AtWRKY6. The transgenic plants overexpressing AtWRKY6 were more susceptible to Pi deficiency and had a similar phenotype as the Atpho1 mutants. This led the researchers to assume the control of AtWRKY6 in the expression of $\mathrm{PHO1}$ and this interaction was indeed proved via ChIP-qPCR analysis. The two W-boxes adjoining the AtPHO1 promoter are responsible for proper binding of AtWRKY 6 through its WRKY domain [106]. It has been suggested that AtWRKY75 and AtWRKY6 differentially and cooperatively regulate the responses to $\mathrm{Pi}$ deficiency. AtWRKY6 also acts as a positive mediator of plant responses during boron deficiency $[107,108]$. AtWRKY45 is mainly localized in the nucleus. The concentration of AtWRKY45 peaked in the roots typically facing Pi starvation. AtWRKY45 RNAi plants showed decreased uptake and hence lower accumulation of Pi during Pi starvation when compared to the wild type plants. The AtWRKY45 RNAi plants were also very sensitive to arsenate present in the soil. The expression of Phosphate Transporter 1;1 (PHT1;1) was stimulated in the transgenic lines overexpressing AtWRKY45. These results have depicted AtWRKY45 as a positive regulator in survival against Pi deficiency. An epistatic genetic regulation between AtWRKY45 and PTH1;1 was also reported [109, 110].

Apart from positively regulating the responses to combat Pi starvation, WRKY45 and WRKY65 are also expressed during carbon starvation. Thus, the WRKY proteins encoded by these genes act as TFs to upregulate the expression of downstream stress-responsive genes [107, 108]. Increased sensitivity to sugar starvation was reported in a transgenic Arabidopsis line overexpressing WRKY72 [71]. The Arabidopsis Nucleoside Diphosphate Kinase 3a (NDPK3a) expression is stimulated in presence of sugar and the protein encoded by the gene gets localized in the mitochondria. The mitochondrion is the chief reservoir of ATP produced mainly by the oxidation of sugars. The NDPK3a promoter has two Wboxes which aid in the binding of SUSIBA2 (HvWRKY46) in barley plants $[109,110]$. There are also reports of AtWRKY4 and AtWRKY34 in mediating the expression of NDPK3a. SUSIBA2 also regulates the expression of ISO1 and Sugar Response Element IIb (SREIIb) in sugar signaling $[109,110]$. Further researches and critical analyses are yet to be made on the queer role of WRKY proteins connecting the plant responses to tackle Pi and sugar starvation. This link may be an indication towards a beneficial symbiotic interaction between relative availability of $\mathrm{Pi}$ in the soil and sugar metabolism $[109,110]$. 
8.5. Radiation Stress. The least studied among all the abiotic stress is the radiation stress and its associated effects on the plant system. However, this field is also gradually gaining enormous importance due to uneven distribution of sunlight throughout the globe. Indiscriminate uses of chlorofluorocarbons (CFCs) and other toxic chemical compounds have led to the formation of the ozone hole. The ozone layer which once acted as an absolute absorber of UV radiations is gradually losing its efficiency. Thus, the plants growing in areas falling under such ozone holes are more prone to UV stress. This is where the significance of designing plants resistant to radiation stress lies.

Radiation stress mainly induces the bleaching of photosynthetic pigments and generation of ROS. Thus, the WRKY genes which encode proteins involved in scavenging of ROS obviously get upregulated. We have already discussed these genes which regulate oxidative stress in plants. However, a WRKY gene OsWRKY89 was identified in rice which has been depicted to regulate responses during UV-B stress (Figure 2). Increased wax deposition on the surfaces of leaves was reported in UV-B stressed transgenic plants overexpressing OsWRKY89 [111]. The increased wax deposition drastically reduced the percentage of UV-B transmittance through the leaves. Further researches are required to create a database on the involvement of multiple WRKY proteins regulating the responses induced by radiation stress.

\section{Conclusion}

In this review, we emphasized the most recent advances on WRKY proteins. WRKY proteins have been thought to have more significance in regulating biotic stress response as compared to abiotic stress. However, modern research works and trailblazing experiments have proved their pivotal roles for developing plant tolerance towards abiotic stress. WRKY proteins have several interacting partners which together coordinate multiple signal cascades. The relation between MAPKs and WRKY proteins also indicates a cooperative signal transduction cascade. The ABA-inducible genes involved in abiotic stress responses are also dependent on their activation by MAPKs or sometimes Calcium Dependent Protein Kinases (CDPKs). A potential cross talk between the MAPKs activating WRKY TFs and those upregulating the abiotic stress response gene is yet to be reported. Improved technologies along with molecular, computational, and informational agrobiology may furnish further details in this respect in near future. Future prospects in this field also include the possibility of a cross talk between abiotic and biotic stress responses mediated by WRKY factors. The structurally related proteins AtWRKY18, AtWRKY40, and AtWRKY60 have provided some hint towards the occurrence of such cross talk (Figure 3). This is because these proteins are participants in the pathways regulated by the three major phytohormones of plant system, that is, SA, JA, and ABA [14]. While SA and JA are involved in transducing response against biotic stress, ABA is an essential proponent in the abiotic stress pathways. WRKY18, WRKY40, and WRKY60 also act as an activator of the IaaH (indole-3-acetamide hydrolase) and IaaM (tryptophan monooxygenase) genes of the T-DNA

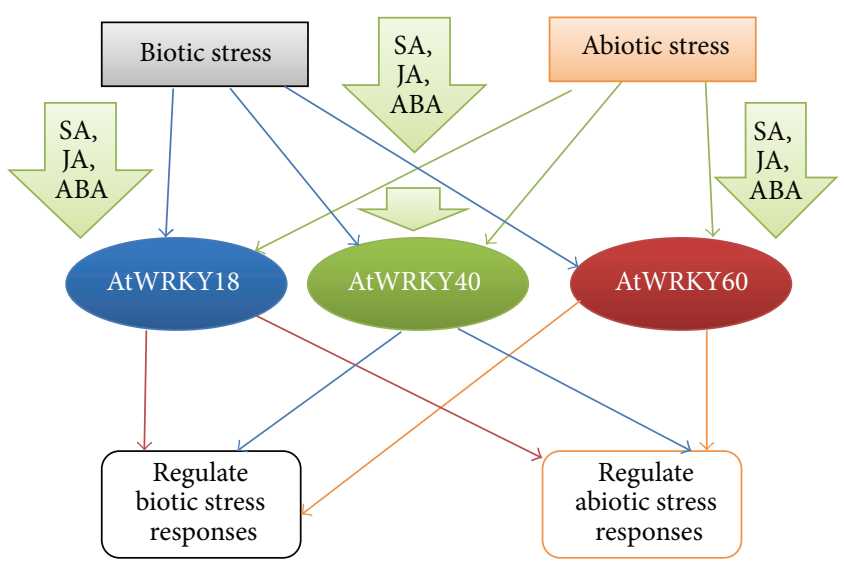

FIgURE 3: Specific WRKY proteins like AtWRKY18, AtWRKY40, and AtWRKY60 have been depicted as mediators of cross talk between plant responses against abiotic and biotic stresses. It has been reported that these proteins get accumulated in response to $\mathrm{SA}$ and JA during biotic stress as well as ABA during abiotic stress responses.

after its integration in the plant following Agrobacterium infection. Thus, WRKYs play a role in the expression of the oncogenes and inducing crown gall disease [112]. In Populus tomentosa, overexpression of Group IIa WRKY gene, PtoWRKY60, resulted in the upregulation of the PR5.1, PR5.2, PR5.4, PR5.5, and other defense-associated genes [113]. Double mutants of WRKY18 and WRKY40 enhanced Arabidopsis resistance against powdery mildew fungus, Golovinomyces orontii by transcriptional reprogramming, alterations in the SA or JA signalling, and Enhanced Disease Susceptibility1 (EDS1) expression, along with accumulation of camalexin. It was further hypothesised that this fungus required the two WRKY proteins for successful infection [114, 115]. Another instance of a cross talk between abiotic and biotic stress responses is the function of AtWRKY25 and AtWRKY33. These proteins show accumulation both under abiotic and biotic stresses. Abiotic stresses like $\mathrm{NaCl}$ and high temperature or pathogenic invasion by Pseudomonas syringae induced the expression of AtWRKY25 and AtWRKY33 [116]. Future prospects also involve the possible identification of specific WRKY proteins which can develop plant tolerance against multiple abiotic stresses. An example of a WRKY protein in this context is the AtWRKY8 which upregulates the plant tolerance against salinity, drought, and also oxidative stress (Figure 2). Genetic engineers can target such single WRKY factors and design multistress tolerant transgenic plant lines. Such tolerant lines can be designed in cereal food crops like rice. Seeds of such crops can be distributed to farmers across wide geographical areas for compatible growth even under harsh environmental conditions.

\section{Conflict of Interests}

The authors declare that there is no conflict of interests regarding the publication of this paper. 


\section{Acknowledgments}

Financial assistance from Science and Engineering Research Board (SERB), Department of Science and Technology, Government of India, through the research grant (SR/FT/LS65/2010) and from Council of Scientific and Industrial Research (CSIR), Government of India, through the research grant (38(1387)/14/EMR-II) to Dr. Aryadeep Roychoudhury is gratefully acknowledged.

\section{References}

[1] A. Matsui, J. Ishida, T. Morosawa et al., “Arabidopsis transcriptome analysis under drought, cold, high-salinity and ABA treatment conditions using a tiling array," Plant and Cell Physiology, vol. 49, no. 8, pp. 1135-1149, 2008.

[2] V. Chinnusamy, K. Schumaker, and J.-K. Zhu, "Molecular genetic perspectives on cross-talk and specificity in abiotic stress signalling in plants," Journal of Experimental Botany, vol. 55, no. 395, pp. 225-236, 2004.

[3] A. RoyChoudhury, B. Gupta, and D. N. Sengupta, "Trans-acting factor designated OSBZ8 interacts with both typical abscisic acid responsive elements as well as abscisic acid responsive element-like sequences in the vegetative tissues of indica rice cultivars," Plant Cell Reports, vol. 27, no. 4, pp. 779-794, 2008.

[4] D. M. Riaño-Pachón, S. Ruzicic, I. Dreyer, and B. MuellerRoeber, "PlnTFDB: an integrative plant transcription factor database," BMC Bioinformatics, vol. 8, article 42, 2007.

[5] A. Ferro, Ed., Abiotic Stress: Role in Sustainable Agriculture, Detrimental Effects and Management Strategies, Environmental Health-Physical, Chemical and Biological Factors, Nova Science, New York, NY, USA, 2014.

[6] M. Akhtar, A. Jaiswal, G. Taj, J. P. Jaiswal, M. I. Qureshi, and N. K. Singh, "DREB1/CBF transcription factors: their structure, function and role in abiotic stress tolerance in plants," Journal of Genetics, vol. 91, no. 3, pp. 385-395, 2012.

[7] P. A. Palmeros-Suarez and J. P. Delano-Frier, "The pivotal role played by transcription factors, small RNAs and epigenetic modifications in the regulation of abiotic stress responses in plants," in Abiotic Stress, A. Ferro, Ed., pp. 1-42, Nova Science Publishers, New York, NY, USA, 2014.

[8] A. Roychoudhury and A. Paul, "Abscisic acid-inducible genes during salinity and drought stress," in Advances in Medicine and Biology, L. V. Berhardt, Ed., vol. 51, pp. 1-78, Nova Science, New York, NY, USA, 2012.

[9] N. Chen, Q. Yang, L. Pan et al., "Identification of 30 MYB transcription factor genes and analysis of their expression during abiotic stress in peanut (Arachis hypogaea L.)," Gene, vol. 533, no. 1, pp. 332-345, 2014.

[10] K. Nakashima, H. Takasaki, J. Mizoi, K. Shinozaki, and K. Yamaguchi-Shinozaki, "NAC transcription factors in plant abiotic stress responses," Biochimica et Biophysica Acta, vol. 1819, no. 2, pp. 97-103, 2012.

[11] S. Ishiguro and K. Nakamura, "Characterization of a cDNA encodine a novel DNA-binding protein, SPF1, that recognizes SP8 sequences in the $5^{\prime}$ upstream regions of genes coding for sporamin and $\beta$-amylase from sweet potato," Molecular and General Genetics, vol. 244, no. 6, pp. 563-571, 1994.

[12] C. P. Marquez and E. J. Pritham, "Phantom, a new subclass of Mutator DNA transposons found in insect viruses and widely distributed in animals," Genetics, vol. 185, no. 4, pp. 1507-1517, 2010 .
[13] J. Li, Role of WRKY Transcription Factors in Arabidopsis Development and Stress Responses, Helsinki University Printing House, Helsinki, Finland, 2014.

[14] L. Chen, Y. Song, S. Li, L. Zhang, C. Zou, and D. Yu, "The role of WRKY transcription factors in plant abiotic stresses," Biochimica et Biophysica Acta: Gene Regulatory Mechanisms, vol. 1819, no. 2, pp. 120-128, 2012.

[15] Z. Zou, "Genome-wide identification and phylogenetic analysis of WRKY transcription factor family in castor bean (Ricinus communis L.)," Chinese Journal of Oil Crop Sciences, vol. 35, no. 1, pp. 36-42, 2013.

[16] K.-F. Wei, J. Chen, Y.-F. Chen, L.-J. Wu, and D.-X. Xie, "Molecular phylogenetic and expression analysis of the complete WRKY transcription factor family in maize," DNA Research, vol. 19, no. 2, pp. 153-164, 2012.

[17] J. Ling, W. Jiang, Y. Zhang et al., "Genome-wide analysis of WRKY gene family in Cucumis sativus," BMC Genomics, vol. 12, article 471, 2011.

[18] C. Cai, E. Niu, H. Du, L. Zhao, Y. Feng, and W. Guo, "Genomewide analysis of the WRKY transcription factor gene family in Gossypium raimondii and the expression of orthologs in cultivated tetraploid cotton," The Crop Journal, vol. 2, no. 2-3, pp. 87-101, 2014.

[19] M. Wang, A. Vannozzi, G. Wang et al., "Genome and transcriptome analysis of the grapevine (Vitis vinifera L.) WRKY gene family," Horticulture Research, vol. 1, article 14016, 2014.

[20] K. Yamasaki, T. Kigawa, M. Seki, K. Shinozaki, and S. Yokoyama, "DNA-binding domains of plant-specific transcription factors: structure, function, and evolution," Trends in Plant Science, vol. 18, no. 5, pp. 267-276, 2013.

[21] P. Agarwal, M. P. Reddy, and J. Chikara, "WRKY: its structure, evolutionary relationship, DNA-binding selectivity, role in stress tolerance and development of plants," Molecular Biology Reports, vol. 38, no. 6, pp. 3883-3896, 2011.

[22] T. Eulgem, P. J. Rushton, S. Robatzek, and I. E. Somssich, “The WRKY superfamily of plant transcription factors," Trends in Plant Science, vol. 5, no. 5, pp. 199-206, 2000.

[23] K. S. Alexandrova and B. V. Conger, "Isolation of two somatic embryogenesis-related genes from orchardgrass (Dactylis glomerata)," Plant Science, vol. 162, no. 2, pp. 301-307, 2002.

[24] M. Lagacé and D. P. Matton, "Characterization of a WRKY transcription factor expressed in late torpedo-stage embryos of Solanum chacoense," Planta, vol. 219, no. 1, pp. 185-189, 2004.

[25] T. Ishida, S. Hattori, R. Sano et al., "Arabidopsis TRANSPARENT TESTA GLABRA2 is directly regulated by R2R3 MYB transcription factors and is involved in regulation of GLABRA2 transcription in epidermal differentiation," The Plant Cell, vol. 19, no. 8, pp. 2531-2543, 2007.

[26] P. J. Rushton, I. E. Somssich, P. Ringler, and Q. J. Shen, "WRKY transcription factors," Trends in Plant Science, vol. 15, no. 5, pp. $247-258,2010$.

[27] J. W. Borrone, D. N. Kuhn, and R. J. Schnell, "Isolation, characterization, and development of WRKY genes as useful genetic markers in Theobroma cacao," Theoretical and Applied Genetics, vol. 109, no. 3, pp. 495-507, 2004.

[28] Y. Qiu, S. J. Jing, J. Fu, L. Li, and D. Yu, "Cloning and analysis of expression profile of 13 WRKY genes in rice," Chinese Science Bulletin, vol. 49, no. 20, pp. 2159-2168, 2004.

[29] H. L. Wu, Z. F. Ni, Y. Y. Yao, G. G. Guo, and Q. X. Sun, "Cloning and expression profiles of 15 genes encoding WRKY transcription factor in wheat (Triticum aestivem L.)," Progress in Natural Science, vol. 18, no. 6, pp. 697-705, 2008. 
[30] Y. Q. Jiang and M. K. Deyholos, "Comprehensive transcriptional profiling of $\mathrm{NaCl}$-stressed Arabidopsis roots reveals novel classes of responsive genes," BMC Plant Biology, vol. 6, article $25,2006$.

[31] X. Xu, C. Chen, B. Fan, and Z. Chen, "Physical and functional interactions between pathogen-induced Arabidopsis WRKY18, WRKY40, and WRKY60 transcription factors," The Plant Cell, vol. 18, no. 5, pp. 1310-1326, 2006.

[32] F. Turck, A. Zhou, and I. E. Somssich, "Stimulus-dependent, promoter-specific binding of transcription factor WRKY1 to its native promoter and the defense-related gene PcPR1-1 in parsley," The Plant Cell, vol. 16, no. 10, pp. 2573-2585, 2004.

[33] G. Mao, X. Meng, Y. Liu, Z. Zheng, Z. Chen, and S. Zhang, "Phosphorylation of a WRKY transcription factor by two pathogen-responsive MAPKs drives phytoalexin biosynthesis in Arabidopsis," The Plant Cell, vol. 23, no. 4, pp. 1639-1653, 2011.

[34] S. J. Li, Q. T. Fu, L. G. Chen, W. D. Huang, and D. Q. Yu, "Arabidopsis thaliana WRKY25, WRKY26, and WRKY33 coordinate induction of plant thermotolerance," Planta, vol. 233, no. 6, pp. 1237-1252, 2011.

[35] S. Zhang and D. F. Klessig, "Pathogen-induced MAP kinases in tobacco," Results and Problems in Cell Differentiation, vol. 27, pp. 65-84, 2000.

[36] A. Danquah, A. de Zelicourt, J. Colcombet, and H. Hirt, "The role of $\mathrm{ABA}$ and MAPK signaling pathways in plant abiotic stress responses," Biotechnology Advances, vol. 32, no. 1, pp. 40$52,2014$.

[37] H. Shen, C. Liu, Y. Zhang et al., "OsWRKY30 is activated by MAP kinases to confer drought tolerance in rice," Plant Molecular Biology, vol. 80, no. 3, pp. 241-253, 2012.

[38] Y. Guan, X. Meng, R. Khanna, E. LaMontagne, Y. Liu, and S. Zhang, "Phosphorylation of a WRKY transcription factor by MAPKs is required for pollen development and function in Arabidopsis," PLoS Genetics, vol. 10, no. 5, Article ID e1004384, 2014.

[39] N. Ishihama, H. Adachi, M. Yoshioka, and H. Yoshioka, "In vivo phosphorylation of WRKY transcription factor by MAPK," in Plant MAP Kinases, G. Komis and J. Šamaj, Eds., vol. 1171 of Methods in Molecular Biology, pp. 171-181, Springer, New York, NY, USA, 2014.

[40] G. Li, X. Meng, R. Wang et al., "Dual-level regulation of ACC synthase activity by MPK3/MPK6 cascade and its downstream WRKY transcription factor during ethylene induction in Arabidopsis," PLoS Genetics, vol. 8, no. 6, Article ID e1002767, 2012.

[41] Y. Cheng, Y. Zhou, Y. Yang et al., "Structural and functional analysis of VQ motif-containing proteins in Arabidopsis as interacting proteins of WRKY transcription factors," Plant Physiology, vol. 159, no. 2, pp. 810-825, 2012.

[42] J.-L. Qiu, B. K. Fiil, K. Petersen et al., "Arabidopsis MAP kinase 4 regulates gene expression through transcription factor release in the nucleus," The EMBO Journal, vol. 27, no. 16, pp. 2214-2221, 2008.

[43] Y. Chi, Y. Yang, Y. Zhou et al., "Protein-protein interactions in the regulation of WRKY transcription factors," Molecular Plant, vol. 6, no. 2, pp. 287-300, 2013.

[44] Z. Lai, Y. Li, F. Wang et al., "Arabidopsis sigma factor binding proteins are activators of the WRKY33 transcription factor in plant defense," The Plant Cell, vol. 23, no. 10, pp. 3824-3841, 2011.

[45] P. Pecher, L. Eschen-Lippold, S. Herklotz et al., "The arabidopsis thaliana mitogen-activated protein kinases MPK3 and MPK6 target a subclass of 'VQ-motif'-containing proteins to regulate immune responses," New Phytologist, vol. 203, pp. 592-606, 2014.

[46] R. Alvarez-Venegas, A. A. Abdallat, M. Guo, J. R. Alfano, and Z. Avramova, "Epigenetic control of a transcription factor at the cross section of two antagonistic pathways," Epigenetics, vol. 2, no. 2, pp. 106-113, 2007.

[47] N. Ay, K. Irmler, A. Fischer, R. Uhlemann, G. Reuter, and K. Humbeck, "Epigenetic programming via histone methylation at WRKY53 controls leaf senescence in Arabidopsis thaliana," Plant Journal, vol. 58, no. 2, pp. 333-346, 2009.

[48] J.-N. Wang, J.-F. Kuang, W. Shan et al., "Expression profiles of a banana fruit linker histone $\mathrm{H} 1$ gene MaHIS1 and its interaction with a WRKY transcription factor," Plant Cell Reports, vol. 31, no. 8, pp. 1485-1494, 2012.

[49] K.-C. Kim, Z. Lai, B. Fan, and Z. Chen, "Arabidopsis WRKY38 and WRKY62 transcription factors interact with histone deacetylase 19 in basal defense," The Plant Cell, vol. 20, no. 9, pp. 2357-2371, 2008.

[50] C. Y. Park, J. H. Lee, J. H. Yoo et al., "WRKY group IId transcription factors interact with calmodulin," FEBS Letters, vol. 579, no. 6, pp. 1545-1550, 2005.

[51] I.-F. Chang, A. Curran, R. Woolsey et al., "Proteomic profiling of tandem affinity purified 14-3-3 protein complexes in Arabidopsis thaliana," Proteomics, vol. 9, no. 11, pp. 2967-2985, 2009.

[52] Y. Shang, L. Yan, Z.-Q. Liu et al., “The Mg-chelatase H subunit of Arabidopsis antagonizes a group of WRKY transcription repressors to relieve ABA-responsive genes of inhibition," The Plant Cell, vol. 22, no. 6, pp. 1909-1935, 2010.

[53] Y. H. Shen, J. Godlewski, A. Bronisz et al., "Significance of 14-3-3 self-dimerization for phosphorylation-dependent target binding," Molecular Biology of the Cell, vol. 14, no. 11, pp. 47214733, 2003.

[54] A. Arulpragasam, A. L. Magno, E. Ingley et al., "The adaptor protein 14-3-3 binds to the calcium-sensing receptor and attenuates receptor-mediated Rho kinase signalling," Biochemical Journal, vol. 441, no. 3, pp. 995-1006, 2012.

[55] J. Dong, C. Chen, and Z. Chen, "Expression profiles of the Arabidopsis WRKY gene superfamily during plant defense response," Plant Molecular Biology, vol. 51, no. 1, pp. 21-37, 2003.

[56] Z.-Q. Liu, L. Yan, Z. Wu et al., "Cooperation of three WRKY-domain transcription factors WRKY18, WRKY40 and WRKY60 in repressing two ABA-responsive genes ABI4 and ABI5 in Arabidopsis," Journal of Experimental Botany, vol. 63, no. 18, pp. 6371-6392, 2012.

[57] H. Chen, Z. Lai, J. Shi, Y. Xiao, Z. Chen, and X. Xu, "Roles of Arabidopsis WRKY18, WRKY40 and WRKY60 transcription factors in plant responses to abscisic acid and abiotic stress," BMC Plant Biology, vol. 10, article 281, 2010.

[58] R. Ramamoorthy, S.-Y. Jiang, N. Kumar, P. N. Venkatesh, and S. Ramachandran, "A comprehensive transcriptional profiling of the WRKY gene family in rice under various abiotic and phytohormone treatments," Plant and Cell Physiology, vol. 49, no. 6, pp. 865-879, 2008.

[59] Z. Xie, Z.-L. Zhang, X. Zou, G. Yang, S. Komatsu, and Q. J. Shen, "Interactions of two abscisic-acid induced WRKY genes in repressing gibberellin signaling in aleurone cells," The Plant Journal, vol. 46, no. 2, pp. 231-242, 2006.

[60] R. Peng, Q. Yao, A. Xiong et al., "A new rice zinc-finger protein binds to the O2S box of the $\alpha$-amylase gene promoter," European Journal of Biochemistry, vol. 271, no. 14, pp. 2949-2955, 2004. 
[61] X. Zou, J. R. Seemann, D. Neuman, and Q. J. Shen, "A WRKY gene from creosote bush encodes an activator of the abscisic acid signaling pathway," The Journal of Biological Chemistry, vol. 279, no. 53, pp. 55770-55779, 2004.

[62] Y. Jiang, G. Liang, and D. Yu, "Activated expression of WRKY57 confers drought tolerance in Arabidopsis," Molecular Plant, vol. 5, no. 6, pp. 1375-1388, 2012.

[63] Z. Tao, Y. Kou, H. Liu, X. Li, J. Xiao, and S. Wang, “OsWRKY45 alleles play different roles in abscisic acid signalling and salt stress tolerance but similar roles in drought and cold tolerance in rice," Journal of Experimental Botany, vol. 62, no. 14, pp. 48634874, 2011.

[64] Z. Xie, P. Ruas, and Q. J. Shen, "Regulatory networks of the phytohormone abscisic acid," Vitamins and Hormones, vol. 72, pp. 235-269, 2005.

[65] D. L. Rushton, P. Tripathi, R. C. Rabara et al., "WRKY transcription factors: key components in abscisic acid signalling," Plant Biotechnology Journal, vol. 10, no. 1, pp. 2-11, 2012.

[66] S. Basu and A. Roychoudhury, "Expression profiling of abiotic stress-inducible genes in response to multiple stresses in rice (Oryza saltiva L.) varieties with contrasting level of stress tolerance," BioMed Research International, vol. 2014, Article ID 706890, 12 pages, 2014.

[67] L. Chen, L. Zhang, D. Li, F. Wang, and D. Yu, "WRKY8 transcription factor functions in the TMV-cg defense response by mediating both abscisic acid and ethylene signaling in Arabidopsis," Proceedings of the National Academy of Sciences of the United States of America, vol. 110, no. 21, pp. E1963-E1971, 2013.

[68] X. Luo, X. Sun, B. Liu et al., "Ectopic expression of a WRKY homolog from Glycine soja alters flowering time in Arabidopsis," PLoS ONE, vol. 8, no. 8, Article ID e73295, 2013.

[69] D. Golldack, I. Lüking, and O. Yang, "Plant tolerance to drought and salinity: stress regulating transcription factors and their functional significance in the cellular transcriptional network," Plant Cell Reports, vol. 30, no. 8, pp. 1383-1391, 2011.

[70] X. Wu, Y. Shiroto, S. Kishitani, Y. Ito, and K. Toriyama, "Enhanced heat and drought tolerance in transgenic rice seedlings overexpressing OsWRKY11 under the control of HSP101 promoter," Plant Cell Reports, vol. 28, no. 1, pp. 21-30, 2009.

[71] Y. Song, L. G. Chen, L. P. Zhang, and D. Q. Yu, “Overexpression of OsWRKY72 gene interferes in the abscisic acid signal and auxin transport pathway of Arabidopsis," Journal of Biosciences, vol. 35, no. 3, pp. 459-471, 2010.

[72] Q.-Y. Zhou, A.-G. Tian, H.-F. Zou et al., "Soybean WRKYtype transcription factor genes, GmWRKY13, GmWRKY21, and GmWRKY54, confer differential tolerance to abiotic stresses in transgenic Arabidopsis plants," Plant Biotechnology Journal, vol. 6, no. 5, pp. 486-503, 2008.

[73] W. Wei, Y. X. Zhang, L. Han, Z. Q. Guan, and T. Y. Chai, “A novel WRKY transcriptional factor from Thlaspi caerulescens negatively regulates the osmotic stress tolerance of transgenic tobacco," Plant Cell Reports, vol. 27, no. 4, pp. 795-803, 2008.

[74] W. B. Jiang and D. Q. Yu, "Arabidopsis WRKY2 transcription factor may be involved inosmotic stress response," Acta Botanica Yunnanica, vol. 31, no. 5, pp. 427-432, 2009.

[75] K. C. Babitha, S. V. Ramu, V. Pruthvi, P. Mahesh, K. N. Nataraja, and M. Udayakumar, "Co-expression of AtbHLH17 and AtWRKY 28 confers resistance to abiotic stress in Arabidopsis," Transgenic Research, vol. 22, no. 2, pp. 327-341, 2013.
[76] Y. Wang, F. Dang, Z. Liu et al., "CaWRKY58, encoding a group I WRKY transcription factor of Capsicum annuum, negatively regulates resistance to Ralstonia solanacearum infection," Molecular Plant Pathology, vol. 14, no. 2, pp. 131-144, 2013.

[77] C.-F. Niu, W. Wei, Q.-Y. Zhou et al., "Wheat WRKY genes TaWRKY2 and TaWRKY19 regulate abiotic stress tolerance in transgenic Arabidopsis plants," Plant, Cell and Environment, vol. 35, no. 6, pp. 1156-1170, 2012.

[78] M. Şahin-Çevik, "A WRKY transcription factor gene isolated from Poncirus trifoliata shows differential responses to cold and drought stresses," Plant Omics Journal, vol. 5, no. 5, pp. 438-445, 2012.

[79] Z. J. Ding, J. Y. Yan, X. Y. Xu et al., "Transcription factor WRKY46 regulates osmotic stress responses and stomatal movement independently in Arabidopsis," Plant Journal, vol. 79, no. 1, pp. 13-27, 2014.

[80] P. Agarwal, M. Dabi, and P. K. Agarwal, "Molecular cloning and characterization of a group II WRKY transcription factor from Jatropha curcas, an important biofuel crop," DNA and Cell Biology, vol. 33, no. 8, pp. 503-513, 2014.

[81] H. Yan, H. Jia, X. Chen, L. Hao, H. An, and X. Guo, "The cotton WRKY transcription factor GhWRKY17 functions in drought and salt stress in transgenic Nicotiana benthamiana through ABA signaling and the modulation of reactive oxygen species production," Plant and Cell Physiology, vol. 55, no. 12, pp. 2060 2076, 2014.

[82] K. Das and A. Roychoudhury, "Reactive oxygen species (ROS) and response of antioxidants as ROS-scavengers during environmental stress in plants," Frontiers in Environmental Science, vol. 2, article 53, 13 pages, 2014.

[83] A. Banerjee and A. Roychoudhury, "Metabolic engineering of lipids in plants," Journal of Plant Science and Research, vol. 1, pp. $1-20,2014$.

[84] Y. Jiang, Y. Duan, J. Yin et al., "Genome-wide identification and characterization of the Populus WRKY transcription factor family and analysis of their expression in response to biotic and abiotic stresses," Journal of Experimental Botany, vol. 65, no. 22, pp. 6629-6644, 2014.

[85] A. Roychoudhury and K. Das, "Functional role of polyamines and polyamine-metabolizing enzymes during salinity, drought and cold stresses," in Plant Adaptation to Environmental Change, N. A. Anjum, S. S. Gill, and R. Gill, Eds., CAB International, London, UK, 2014.

[86] L. G. Chen, L. P. Zhang, and D. Q. Yu, "Wounding-induced WRKY8 is involved in basal defense in Arabidopsis," Molecular Plant-Microbe Interactions, vol. 23, no. 5, pp. 558-565, 2010.

[87] O. van Aken, B. Zhang, S. Law, R. Narsai, and J. Whelan, "AtWRKY40 and AtWRKY63 modulate the expression of stress-responsive nuclear genes encoding mitochondrial and chloroplast proteins," Plant Physiology, vol. 162, no. 1, pp. 254271, 2013.

[88] B. Zhang, C. Carrie, A. Ivanova et al., "LETM proteins play a role in the accumulation of mitochondrially encoded proteins in Arabidopsis thaliana and AtLETM2 displays parent of origin effects," The Journal of Biological Chemistry, vol. 287, no. 50, pp. 41757-41773, 2012.

[89] S. Ciftci-Yilmaz, M. R. Morsy, L. Song et al., "The EAR-motif of the Cys2/His2-type zinc finger protein Zat7 plays a key role in the defense response of Arabidopsis to salinity stress," Journal of Biological Chemistry, vol. 282, no. 12, pp. 9260-9268, 2007.

[90] L. Rizhsky, S. Davletova, H. Liang, and R. Mittler, "The zinc finger protein Zat12 is required for Cytosolic Ascorbate Peroxidase 
1 expression during oxidative stress in Arabidopsis," The Journal of Biological Chemistry, vol. 279, no. 12, pp. 11736-11743, 2004.

[91] T. E. Scarpeci, M. I. Zanor, N. Carrillo, B. Mueller-Roeber, and E. M. Valle, "Generation of superoxide anion in chloroplasts of Arabidopsis thaliana during active photosynthesis: a focus on rapidly induced genes," Plant Molecular Biology, vol. 66, no. 4, pp. 361-378, 2008.

[92] S. Vanderauwera, K. Vandenbroucke, A. Inzé et al., “AtWRKY15 perturbation abolishes the mitochondrial stress response that steers osmotic stress tolerance in Arabidopsis," Proceedings of the National Academy of Sciences of the United States of America, vol. 109, no. 49, pp. 20113-20118, 2012.

[93] S. Rosenvasser, S. Mayak, and H. Friedman, "Increase in reactive oxygen species (ROS) and in senescence-associated gene transcript (SAG) levels during dark-induced senescence of Pelargonium cuttings, and the effect of gibberellic acid," Plant Science, vol. 170, no. 4, pp. 873-879, 2006.

[94] M. Han, C.-Y. Kim, J. Lee, S.-K. Lee, and J.-S. Jeon, “OsWRKY42 represses OsMTld and induces reactive oxygen species and leaf senescence in rice," Molecules and Cells, vol. 37, no. 7, pp. 532539, 2014.

[95] C. Wang, P. Deng, L. Chen et al., "A wheat WRKY transcription factor TaWRKY10 confers tolerance to multiple abiotic stresses in transgenic tobacco," PLoS ONE, vol. 8, no. 6, Article ID e65120, 2013.

[96] S. J. Li, X. Zhou, L. G. Chen, W. D. Huang, and D. Q. Yu, "Functional characterization of Arabidopsis thaliana WRKY39 in heat stress," Molecules and Cells, vol. 29, no. 5, pp. 475-483, 2010.

[97] W. Busch, M. Wunderlich, and F. Schöffl, "Identification of novel heat shock factor-dependent genes and biochemical pathways in Arabidopsis thaliana," Plant Journal, vol. 41, no. 1, pp. 1-14, 2005.

[98] C. Zou, W. Jiang, and D. Yu, "Male gametophyte-specific WRKY34 transcription factor mediates cold sensitivity of mature pollen in Arabidopsis," Journal of Experimental Botany, vol. 61, no. 14, pp. 3901-3914, 2010.

[99] C. Marè, E. Mazzucotelli, C. Crosatti, E. Francia, A. M. Stanca, and L. Cattivelli, "Hv-WRKY38: a new transcription factor involved in cold- and drought-response in barley," Plant Molecular Biology, vol. 55, no. 3, pp. 399-416, 2004.

[100] L. Wang, W. Zhu, L. Fang et al., "Genome-wide identification of WRKY family genes and their response to cold stress in Vitis vinifera," BMC Plant Biology, vol. 14, no. 1, article 103, 2014.

[101] T. Huang and J. G. Duman, "Cloning and characterization of a thermal hysteresis (antifreeze) protein with DNA-binding activity from winter bittersweet nightshade, Solanum dulcamara," Plant Molecular Biology, vol. 48, no. 4, pp. 339-350, 2002.

[102] F. Wang, X. L. Hou, J. Tang et al., "A novel cold-inducible gene from Pak-choi (Brassica campestris ssp. chinensis), BcWRKY46, enhances the cold, salt and dehydration stress tolerance in transgenic tobacco," Molecular Biology Reports, vol. 39, no. 4, pp. 4553-4564, 2012.

[103] U. K. S. Shekhawat, T. R. Ganapathi, and L. Srinivas, "Cloning and characterization of a novel stress-responsive WRKY transcription factor gene (MusaWRKY71) from Musa spp. cv. Karibale Monthan (ABB group) using transformed banana cells," Molecular Biology Reports, vol. 38, no. 6, pp. 4023-4035, 2011.

[104] N. Yokotani, Y. Sato, S. Tanabe et al., "WRKY76 is a rice transcriptional repressor playing opposite roles in blast disease resistance and cold stress tolerance," Journal of Experimental Botany, vol. 64, no. 16, pp. 5085-5097, 2013.

[105] B. N. Devaiah, A. S. Karthikeyan, and K. G. Raghothama, "WRKY75 transcription factor is a modulator of phosphate acquisition and root development in Arabidopsis," Plant Physiology, vol. 143, no. 4, pp. 1789-1801, 2007.

[106] Y.-F. Chen, L.-Q. Li, Q. Xu, Y.-H. Kong, H. Wang, and W.-H. Wu, "The WRKY6 transcription factor modulates PHOSPHATE1 expression in response to low pi stress in Arabidopsis," Plant Cell, vol. 21, no. 11, pp. 3554-3566, 2009.

[107] I. Kasajima, Y. Ide, M. Yokota Hirai, and T. Fujiwara, "WRKY6 is involved in the response to boron deficiency in Arabidopsis thaliana," Physiologia Plantarum, vol. 139, no. 1, pp. 80-92, 2010.

[108] A. L. Contento, S.-J. Kim, and D. C. Bassham, "Transcriptome profiling of the response of Arabidopsis suspension culture cells to Suc starvation," Plant Physiology, vol. 135, no. 4, pp. 2330 2347, 2004.

[109] H. Wang, Q. Xu, Y. H. Kong et al., “Arabidopsis WRKY45 transcription factor activates PHOSPHATE TRANSPORTER1;1 expression in response to phosphate starvation," Plant Physiology, vol. 164, no. 4, pp. 2020-2029, 2014.

[110] M. Bakshi and R. Oelmüller, "WRKY transcription factors: Jack of many trades in plants," Plant Signaling and Behavior, vol. 9, Article ID e27700, 2014.

[111] H. Wang, J. Hao, X. Chen et al., "Overexpression of rice WRKY89 enhances ultraviolet $\mathrm{B}$ tolerance and disease resistance in rice plants," Plant Molecular Biology, vol. 65, no. 6, pp. 799-815, 2007.

[112] Y. Zhang, C.-W. Lee, N. Wehner et al., "Regulation of oncogene expression in T-DNA-transformed plant cells," PLoS Pathogens, vol. 11, no. 1, Article ID e1004620, 2015.

[113] S. Ye, Y. Jiang, Y. Duan et al., "Constitutive expression of the poplar WRKY transcription factor PtoWRKY60 enhances resistance to Dothiorella gregaria Sacc. in transgenic plants," Tree Physiology, vol. 34, no. 10, pp. 1118-1129, 2014.

[114] M. Schön, A. Töller, C. Diezel et al., "Analyses of wrky18 wrky40 plants reveal critical roles of SA/EDS1 signaling and indoleglucosinolate biosynthesis for Golovinomyces orontii resistance and a loss-of resistance towards Pseudomonas syringae pv. tomato AvrRPS4," Molecular Plant-Microbe Interactions, vol. 26, no. 7, pp. 758-767, 2013.

[115] S. P. Pandey, M. Roccaro, M. Schön, E. Logemann, and I. E. Somssich, "Transcriptional reprogramming regulated by WRKY18 and WRKY40 facilitates powdery mildew infection of Arabidopsis," The Plant Journal, vol. 64, no. 6, pp. 912-923, 2010.

[116] Z. Zheng, S. L. Mosher, B. Fan, D. F. Klessig, and Z. Chen, "Functional analysis of Arabidopsis WRKY25 transcription factor in plant defense against Pseudomonas syringae," BMC Plant Biology, vol. 7, article 2, 2007.

[117] Y. Zhang and L. Wang, "The WRKY transcription factor superfamily: its origin in eukaryotes and expansion in plants," BMC Evolutionary Biology, vol. 5, pp. 1-12, 2005.

[118] Z. Xie, Z.-L. Zhang, X. Zou et al., "Annotations and functional analyses of the rice WRKY gene superfamily reveal positive and negative regulators of abscisic acid signaling in aleurone cells," Plant Physiology, vol. 137, no. 1, pp. 176-189, 2005.

[119] H. Chen, Z. B. Lai, J. W. Shi, Y. Xiao, Z. X. Chen, and X. P. $\mathrm{Xu}$, "Roles of Arabidopsis WRKY18, WRKY40 and WRKY60 transcription factors in plant responses to abscisic acid and abiotic stress," BMC Plant Biology, vol. 10, article 281, 2010. 
[120] Y. Song, S. J. Jing, and D. Q. Yu, "Overexpression of the stress-induced OsWRKY08 improves osmotic stress tolerance in Arabidopsis," Chinese Science Bulletin, vol. 54, no. 24, pp. 4671-4678, 2009.

[121] X. Wu, Y. Shiroto, S. Kishitani, Y. Ito, and K. Toriyama, "Enhanced heat and drought tolerance in transgenic rice seedlings overexpressing OsWRKY11 under the control of HSP101 promoter," Plant Cell Reports, vol. 28, no. 1, pp. 21-30, 2009.

[122] H. Wang, J. J. Hao, X. J. Chen et al., "Overexpression of rice WRKY89 enhances ultraviolet $\mathrm{B}$ tolerance and disease resistance in rice plants," Plant Molecular Biology, vol. 65, no. 6, pp. 799-815, 2007.

[123] Y. P. Qiu and D. Q. Yu, "Over-expression of the stress-induced OsWRKY45 enhances disease resistance and drought tolerance in Arabidopsis," Environmental and Experimental Botany, vol. 65, no. 1, pp. 35-47, 2009.

[124] S. Yu, C. Ligang, Z. Liping, and Y. Diqiu, "Overexpression of OsWRKY72 gene interferes in the abscisic acid signal and auxin transport pathway of Arabidopsis," Journal of Biosciences, vol. 35, no. 3, pp. 459-471, 2010.

[125] N. Ishihama and H. Yoshioka, "Post-translational regulation of WRKY transcription factors in plant immunity," Current Opinion in Plant Biology, vol. 15, no. 4, pp. 431-437, 2012.

[126] Y. Hu, Q. Dong, and D. Yu, “Arabidopsis WRKY46 coordinates with WRKY70 and WRKY53 in basal resistance against pathogen Pseudomonas syringae," Plant Science, vol. 185-186, pp. 288-297, 2012.

[127] T. Wei, B. Ou, J. Li et al., "Transcriptional profiling of rice early response to Magnaporthe oryzae identified OsWRKYs as important regulators in rice blast resistance," PLoS ONE, vol. 8, no. 3, Article ID e59720, 2013.

[128] Z. Tao, H. Liu, D. Qiu et al., "A pair of allelic WRKY genes play opposite roles in rice-bacteria interactions," Plant Physiology, vol. 151, no. 2, pp. 936-948, 2009.

[129] A. Lan, J. Huang, W. Zhao, Y. Peng, Z. Chen, and D. Kang, "A salicylic acid-induced rice (Oryza sativa L.) transcription factor OsWRKY77 is involved in disease resistance of Arabidopsis thaliana," Plant Biology, vol. 15, no. 3, pp. 452-461, 2013.

[130] Y. Meng and R. P. Wise, "HvWRKY10, HvWRKY19, and HvWRKY28 regulate Mla-triggered immunity and basal defense to barley powdery mildew," Molecular Plant-Microbe Interactions, vol. 25, no. 11, pp. 1492-1505, 2012. 

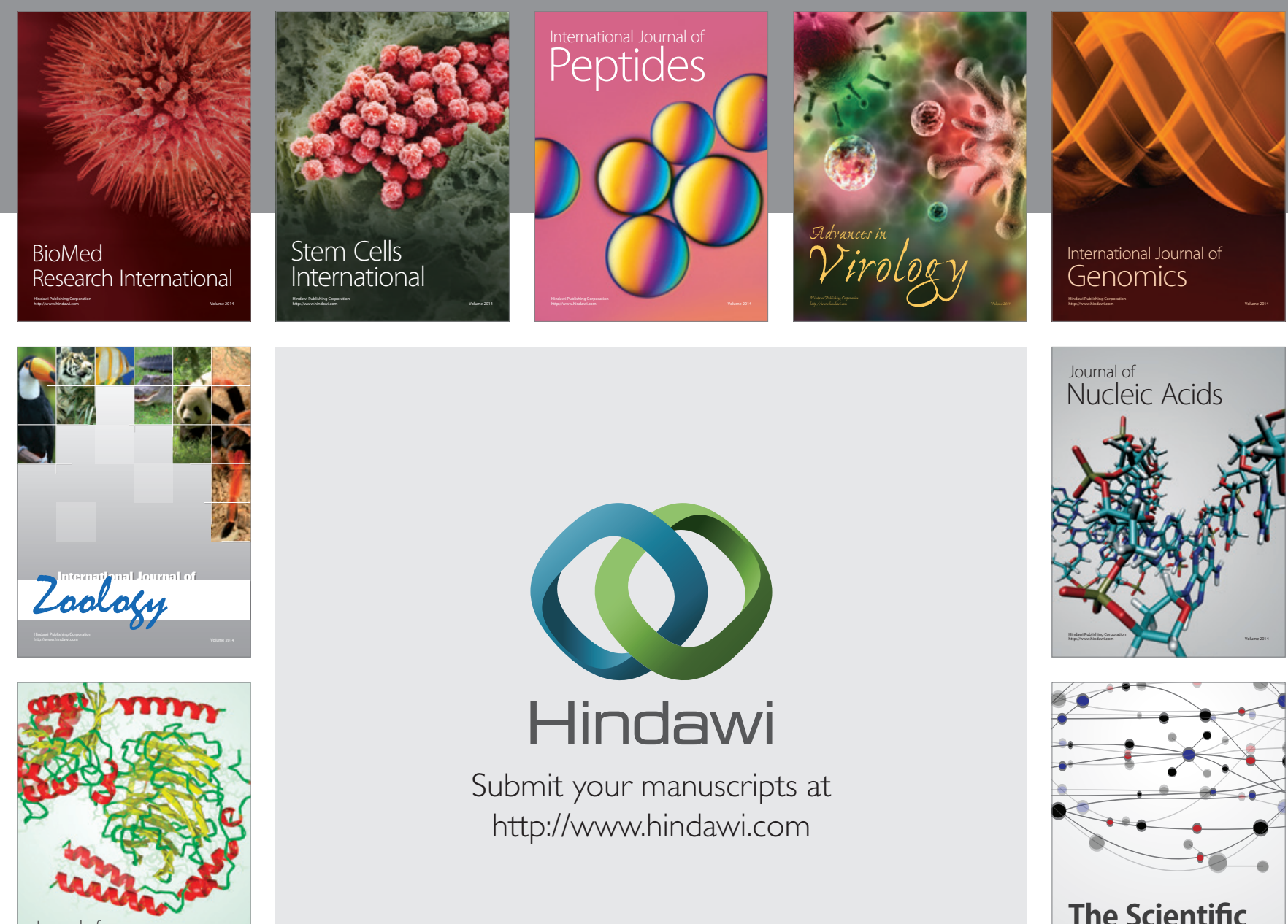

Submit your manuscripts at

http://www.hindawi.com

Journal of
Signal Transduction
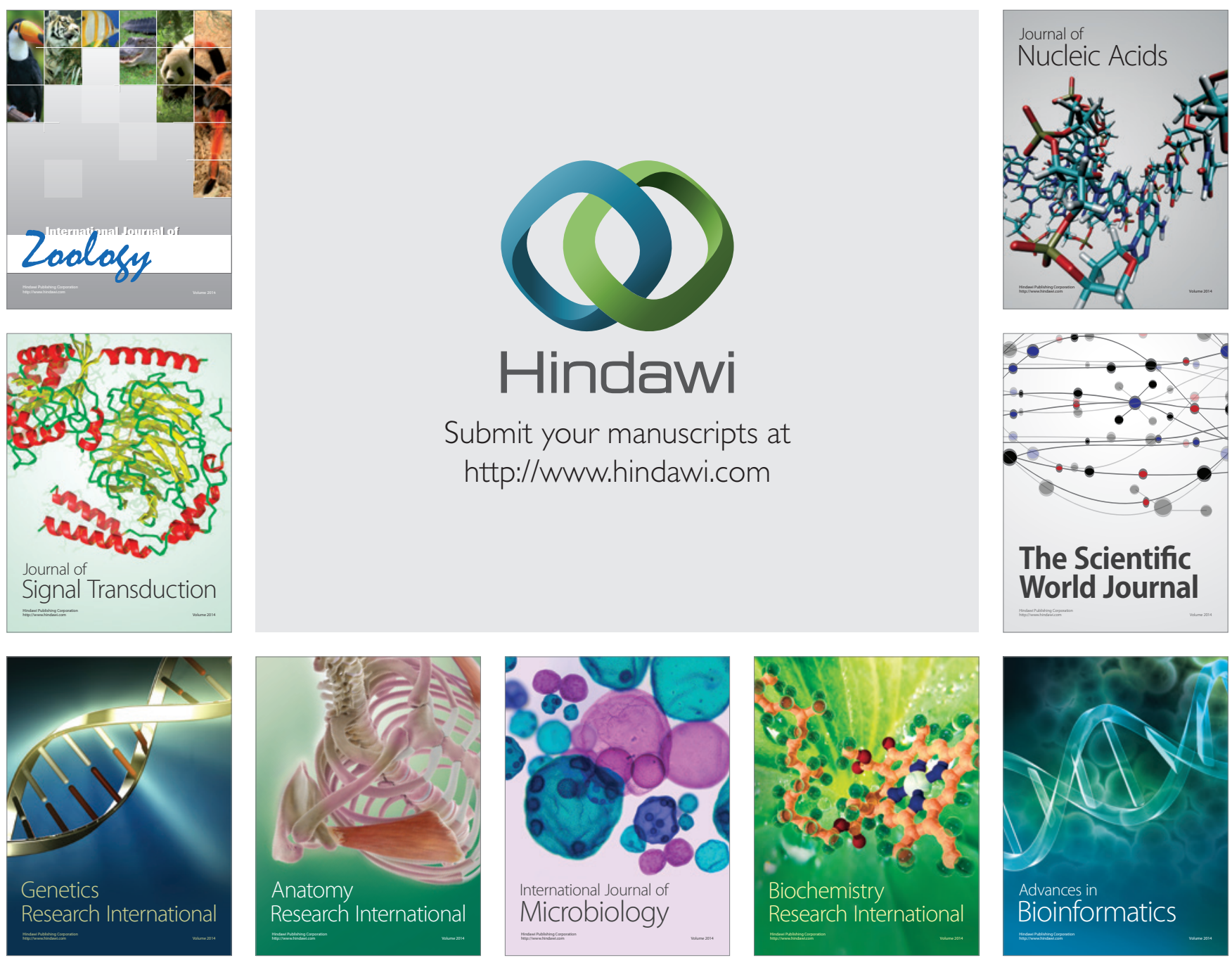

The Scientific World Journal
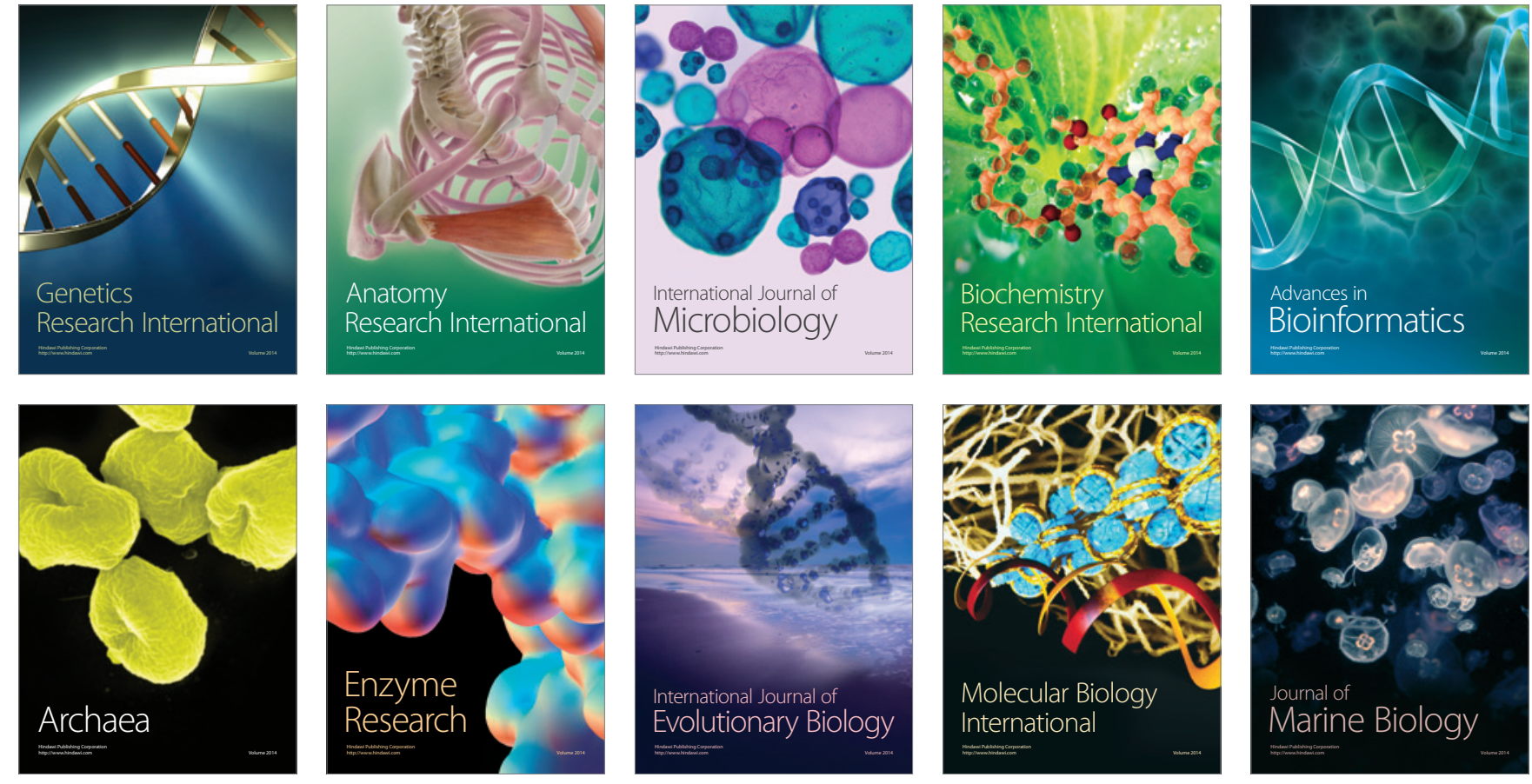